\title{
Mode competition in cylindrical flows driven by sidewall oscillations
}

\author{
C. Panades, F. Marques, ${ }^{*}$ and A. Meseguer \\ Departament de Física Aplicada, Universitat Politècnica de Catalunya, Barcelona 08034, Spain \\ (Received 14 January 2013; published 3 April 2013)
}

\begin{abstract}
The transition from a two-dimensional to three-dimensional flow in systems with spatial $\mathrm{O}(2)$ symmetry and spatiotemporal $\mathrm{Z}_{2}$ symmetry happens in many fluid systems, like wakes or periodically forced flows. In most of these systems, the dynamics after the first bifurcation is very complex and involves cascades of bifurcations in a very narrow parameter range. A numerical study of a flow in an enclosed cylindrical cavity driven by axial oscillations of the sidewall, which allows a detailed study of the secondary bifurcations and the corresponding mode interactions, is presented. The study focuses on a codimension- 2 point that acts as the organizing center of the dynamics for moderate values of the forcing frequency. The unraveled dynamics is very rich, including slow-fast dynamics and hysteresis, and may help understand the bifurcation cascades in more complex systems.
\end{abstract}

DOI: 10.1103/PhysRevE.87.043001

PACS number(s): 47.20.Ib, 47.20.Ky, 05.45.-a

\section{INTRODUCTION}

The transition from two-dimensional to three-dimensional flows is of fundamental interest in fluid dynamics. The term two-dimensional refers to flows whose velocity field only possesses two components that solely depend on the corresponding two coordinates in a convenient coordinate system. Planar two-dimensional flows, like the Von Kármán vortex street, or axisymmetric flows with zero azimuthal velocity are examples of such two-dimensional flows. The time-periodic Kármán vortex street and other bluff-body wakes are invariant in the spanwise direction to both translations and reflections, thus generating the $\mathrm{O}(2)$ symmetry group. Furthermore, these flows have an additional space-time symmetry: a reflection about the wake centerline followed by a half-period temporal evolution. In several wake flows, two distinct synchronous modes that break into the spanwise direction (with real Floquet exponent) have been observed experimentally [1-4], computed as direct instabilities from the flow [2,5-8], and studied theoretically [9-11]. These modes are associated with breaking or preserving the spatiotemporal symmetry. Unfortunately, wake flows can only be studied in terms of a single control parameter; therefore, only one of both synchronous modes can be observed at onset, the other one appearing at secondary bifurcations in the form of mixed modes or complex timedependant flows. Additionally, there are instabilities of the basic periodic flow with complex Floquet multipliers, resulting in quasiperiodic flows, which may also appear in secondary instabilities.

In order to better understand the three-dimensional bifurcated modes, other fluid problems with the same symmetry group [spatial $\mathrm{O}(2)$ symmetry and spatiotemporal $\mathrm{Z}_{2}$ symmetry] have been studied. These problems possess more governing parameters, and by a convenient selection of them, the different modes appear at the first bifurcation. One of these problems is the flow in a rectangular cavity driven by the periodic oscillation of one wall [11-15]. In this case, both the synchronous and the quasiperiodic modes have been obtained and analyzed experimentally and numerically as the primary bifurcation for different control parameters. However,

*francisco.marques@upc.edu as in many wake scenarios, the spanwise direction is not truly periodic but of infinite extent. By expanding in Fourier series, this flow can be made periodic, but the wave number varies continuously, resulting in a continuum of bifurcating modes that interact in a complex manner. Moreover, the presence of end walls in the spanwise direction, no matter how far apart, modifies the properties of the pure periodic modes [15]. Therefore, it would be very convenient to have a flow with the mentioned symmetries and the $\mathrm{SO}(2)$ symmetry fulfilled exactly. Axisymmetric bluff body wakes are an option, like the flow past a ring [16,17]. Unluckily, in this setting the spatiotemporal symmetry is only satisfied in the limit of zero curvature, and the effects of the broken symmetry result in the presence of subharmonic modes that are absent in the presence of the exact symmetry. Similar phenomena occurred in the wakes produced in a square cylinder that has been rotated about its axis [18]. A periodically driven annular cavity solves this issue and has been considered in Ref. [8]. This case is a continuous deformation of the planar driven cavity flow in the sense that the infinite spanwise direction becomes a periodic angular direction. The curvature of the annulus in conjunction with the Reynolds number determines the first mode to become unstable. It is found that even very near the onset of three-dimensional instabilities, the dynamics are dominated by a variety of mixed modes with complicated spatiotemporal structures. Even though the latter problem has the correct symmetries and it is possible to select which one of the three-dimensional modes becomes unstable first, the complexities of the dynamics and the mode interactions right from the onset do not allow a detailed analysis of the bifurcated modes and their interactions. This phenomenon is attributed to the fact that the azimuthal wave numbers of the bifurcated solutions are large, so there are many modes bifurcating in a very narrow parameter range, rendering it impossible to follow the cascade of secondary bifurcations taking place.

In the present study, a problem with the same symmetries such that the azimuthal wave numbers of the bifurcated solutions are small, has been suitably selected. The system consists of a fluid confined in a finite cylinder, driven by axial oscillations of the sidewall. The linear stability analysis of this flow [19] revealed that by an appropriate selection of the forcing frequency both the synchronous and the quasiperiodic modes could be obtained, and three codimension- 2 points 
where two of these different modes bifurcate simultaneously have been found. These codimension- 2 points act as organizing centers of the dynamics, and detailed analysis of the bifurcations and mode interactions around one of this points is presented here, revealing very rich dynamics, including different symmetry-breaking bifurcations, hysteresis, and slow-fast dynamics associated with heteroclinic cycles.

\section{BACKGROUND}

The system under consideration is a Newtonian fluid of kinematic viscosity $v$ enclosed in a cylindrical cavity of radius $R$ and height $h$, whose lateral wall oscillates harmonically in the axial direction, with period $\tau$ and maximum axial velocity $V_{\max }$, while the top and bottom lids remain at rest, as shown schematically in Fig. 1. The radius $R$ and the viscous time $R^{2} / v$ are the length and time scales, respectively. The system is governed by three independent nondimensional parameters $(\Gamma, \mathrm{Re}, \mathrm{St})$. The aspect ratio $\Gamma=h / R$ describes the cylinder geometry and, in the current study, it is kept fixed to $\Gamma=2$. The Reynolds number $\operatorname{Re}=V_{\max } R / v$ measures the amplitude of the forcing, while the Stokes number St $=R^{2} / \nu \tau$ is the inverse of the nondimensional forcing period, $T_{1}=1 / \mathrm{St}$. The flow is governed by the nondimensional Navier-Stokes equations

$$
\left(\frac{\partial}{\partial t}+\mathbf{u} \cdot \nabla\right) \mathbf{u}=-\nabla p+\nabla^{2} \mathbf{u}, \quad \nabla \cdot \mathbf{u}=0
$$

where $\mathbf{u}=(u, v, w)$ is the velocity field in cylindrical coordinates $(r, \theta, z) \in[0,1] \times[0,2 \pi] \times[-\Gamma / 2, \Gamma / 2]$, and $p$ is the kinematic pressure. The vorticity associated to the velocity field is $\nabla \times \mathbf{u}=(\xi, \eta, \zeta)$. No-slip velocity boundary conditions are used on all walls:

$$
\begin{aligned}
\mathbf{u}(r, \theta, \pm \Gamma / 2, t) & =(0,0,0), \\
\mathbf{u}(1, \theta, z, t) & =\left(0,0, \operatorname{Re} \sin \left(2 \pi t / T_{1}\right)\right) .
\end{aligned}
$$

(a)

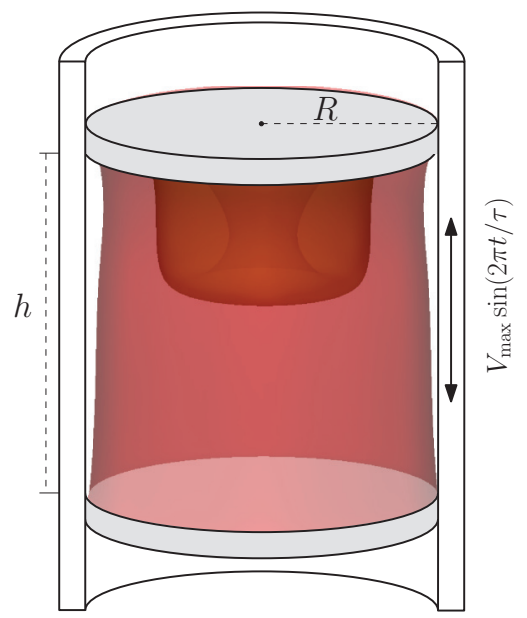

The governing equations have been solved using a secondorder time-splitting method, with spectral spatial discretization, described in detail in Refs. [19,20].

The governing equations and boundary conditions are invariant under the following spatial and spatiotemporal symmetries:

$$
\begin{aligned}
K_{\beta}(u, v, w)(r, \theta, z, t) & =(u,-v, w)(r, 2 \beta-\theta, z, t), \\
R_{\alpha}(u, v, w)(r, \theta, z, t) & =(u, v, w)(r, \theta+\alpha, z, t), \\
H(u, v, w)(r, \theta, z, t) & =(u, v,-w)\left(r, \theta,-z, t+T_{1} / 2\right),
\end{aligned}
$$

for any angles $\alpha$ and $\beta . K_{\beta}$ is the reflection about the meridional plane $\theta=\beta$, and $R_{\alpha}$ is the rotation of angle $\alpha$ about the cylinder axis. $K_{\beta}$ and $R_{\alpha}$ do not commute $\left(K_{\beta} R_{\alpha}=\right.$ $R_{-\alpha} K_{\beta}$ ) and generate the group $\mathrm{O}(2)$ acting on the periodic azimuthal $\theta$ direction. $H$ consists of a reflection about the midplane $z=0$ together with a half-forcing-period evolution in time. This transformation commutes with $\mathrm{O}(2)$ and, acting on the governing equations, its square $H^{2}$ is the identity. Therefore, the complete symmetry group of the problem is $\mathrm{O}(2) \times \mathrm{Z}_{2}^{\mathrm{ST}}$. The action of these symmetries on the vorticity is given by

$$
\begin{aligned}
K_{\beta}(\xi, \eta, \zeta)(r, \theta, z, t) & =(-\xi, \eta,-\zeta)(r, 2 \beta-\theta, z, t), \\
R_{\alpha}(\xi, \eta, \zeta)(r, \theta, z, t) & =(\xi, \eta, \zeta)(r, \theta+\alpha, z, t), \\
H(\xi, \eta, \zeta)(r, \theta, z, t) & =(-\xi,-\eta, \zeta)\left(r, \theta,-z, t+T_{1} / 2\right) .
\end{aligned}
$$

As a consequence, the basic flow, which possesses all the symmetries of the problem, is always axisymmetric and time periodic, synchronous with the forcing. The axial oscillations of the cylinder sidewall produce periodic Stokes-type boundary layers on the oscillating wall. These layers collide with the end walls to form rollers; they are formed every half-period alternately on each end wall. These alternating rollers can be observed in Figs. 1(a) and 1(b), where isosurfaces of axial velocity $w$ half-period apart are plotted. The axial velocity is

FIG. 1. (Color online) Schematic of the apparatus. Panels (a) and (b) display the base state, synchronous and axisymmetric, half a forcing period apart, exhibiting the space-time symmetry $H$. Translucent isosurfaces of axial velocity show the rollers alternating at the end walls, and the sidewall boundary layer. Panel (c) is a snapshot of a synchronous $B_{1}$ bifurcated state. Solid isosurfaces of axial vorticity show the braid structures associated with three-dimensional instabilities of the rollers. Dark and light (red and yellow online) isosurfaces indicate positive and negative values respectively. 
the only nonzero component of the driving mechanisms; the sidewall oscillation and the isosurfaces of $w$ show both the rollers and the sidewall boundary layer structure.

When the basic flow undergoes succesive bifurcations, some of the symmetries are broken, and it is convenient to have a measure of the symmetry breaking. If $\mathrm{SO}(2)$, i.e., the azimuthal rotation symmetries $R_{\alpha}$ are broken, the kinetic energies of the nonzero azimuthal Fourier components of the velocity field [zero for an axisymmetric solution $\mathrm{SO}(2)$ equivariant] give a good measure of the symmetry-breaking process. It will be employed,

$$
E_{n}=\int_{\mathcal{D}} \mathbf{u}_{n} \mathbf{u}_{n}^{*} r d r d z, \quad \mathbf{u}(r, \theta, z, t)=\sum_{n=-N}^{N} \mathbf{u}_{n}(r, z, t) e^{i n \theta},
$$

where the integration domain $\mathcal{D}$ is $(r, z) \in[0,1] \times[-\Gamma / 2, \Gamma / 2]$ and $\mathbf{u}_{n}$ is the $n$th Fourier mode of the velocity field. As the basic flow has zero radial and axial vorticity, any of these vorticity components is also a good measure of the three-dimensional nature of the bifurcated flow. The axial vorticity will be used to visualize 3D solutions, as has been done in the snapshot of a bifurcated state in Fig. 1(c).

A symmetry check for the reflection symmetry $K_{\beta}$ can be implemented as follows. Let $g(r, \theta, z, t)$ be a variable such that

$$
\begin{aligned}
K_{\beta} g(r, \theta, z, t) & =g(r, 2 \beta-\theta, z, t), \\
g(r, \theta, z, t) & =\sum_{n=-N}^{N} G_{n}(r, z, t) e^{i n \theta},
\end{aligned}
$$

for example, the axial velocity or the azimuthal vorticity, with Fourier components $G_{n}$. Since $g$ is real, $G_{-n}=\overline{G_{n}}$. Assuming that $g$ is $K_{\beta}$ invariant,

$$
\begin{aligned}
K_{\beta} g(r, \theta, z, t) & =g(r, \theta, z, t) \Rightarrow e^{2 i n \beta} G_{n}=G_{-n}=\overline{G_{n}} \\
& \Rightarrow e^{i n \beta} G_{n}=\overline{e^{i n \beta} G_{n}} \Rightarrow \arg G_{n}=-n \beta,
\end{aligned}
$$

where $\beta$ can be time dependent, but it is independent of $(r, z)$. In order to define a symmetry parameter, the phases of the Fourier components $G_{n}$ must be examined. Introducing $G_{n}=$ $\left|G_{n}\right| e^{i \phi_{n}}$, the mean phase and the corresponding standard deviation can be easily computed:

$$
\begin{aligned}
\beta_{n}(t) & =\frac{2}{\Gamma} \int_{\mathcal{D}} \phi_{n}(r, z, t) r d r d z, \\
\sigma_{\beta_{n}}^{2}(t) & =\frac{2}{\Gamma} \int_{\mathcal{D}}\left(\phi_{n}(r, z, t)-\beta_{n}\right)^{2} r d r d z .
\end{aligned}
$$

The standard deviation $\sigma_{\beta_{n}}$ is a good measure of the reflection symmetry of the different Fourier modes. For a global measure of this asymmetry, the correlation of the angles of the Fourier modes respect to the first one has to be checked, $\beta_{n}=n \beta_{1}$. Therefore, the symmetry parameter $S_{K_{\beta}}$ is defined as

$$
\begin{aligned}
S_{K_{\beta}}^{2} & =\sum_{n=1}^{N} w_{n}\left[\sin ^{2}\left(\beta_{n}-n \beta_{1}\right)+\sigma_{\beta_{n}}^{2}\right], \\
w_{n} & =\left\|G_{n}\right\|_{\infty} / \sum_{k=2}^{N}\left\|G_{k}\right\|_{\infty},
\end{aligned}
$$

which is always positive, and zero precisely when the symmetry line of mode $n$ coincides with the symmetry line of mode one; in other words, when $g$ is $K_{\beta}$ invariant. Each mode has been weighted according to its contribution to the full solution using the maximum norm $\left\|G_{k}\right\|_{\infty}$ in the domain $\mathcal{D}$.

Last but not least, it remains to give a good measure of the $H$ symmetry. One option is to observe the flow half-period apart in symmetric planes with respect to $z=0$. A more rigorous estimation is

$$
S_{H}=\left\|w(r, \theta, z, t)+w\left(r, \theta,-z, t+T_{1} / 2\right)\right\|_{2},
$$

which is zero for an $H$-symmetric solution, according to (3c). In particular, $S_{H}=0$ means that $w$ is time periodic and can be expanded in Fourier modes:

$$
w(r, \theta, z, t)=\sum_{n} w_{n}(r, \theta, z) e^{2 \pi i n t / T_{1}} .
$$

At the midplane $z=0$, from Eq. (3c) it is obtained that

$$
\begin{aligned}
w(r, \theta, 0, t) & =-w\left(r, \theta, 0, t+T_{1} / 2\right) \Rightarrow w_{n}(r, \theta, 0) \\
& =(-1)^{n+1} w_{n}(r, \theta, 0),
\end{aligned}
$$

and the even temporal Fourier components of the axial velocity on the midplane are zero for an $H$-symmetric solution. As a consequence, the magnitude of the even Fourier components is a suitable measure of the $H$ symmetry breaking, a result useful in the analysis of the solutions.

\section{A. Previous results}

It was observed [19] that by increasing the amplitude of the forcing, $\mathrm{Re}$, beyond a critical value, $\mathrm{Re}_{c}(\mathrm{St})$, the basic state underwent a symmetry-breaking bifurcation yielding bifurcated three-dimensional states. Depending on St, the basic state might undergo either synchronous or Neimark-Sacker bifurcations. The synchronous bifurcations are pitchforks of revolution that can break (mode $A$ ) or preserve (mode $B$ ) the space-time symmetry $H$. The Neimark-Sacker bifurcations result in quasiperiodic solutions that are modulated traveling or standing waves [11]. Figure 2 shows the bifurcation curves in the $(\mathrm{St}, \mathrm{Re})$ space. For small and high $\mathrm{St}$, the synchronous

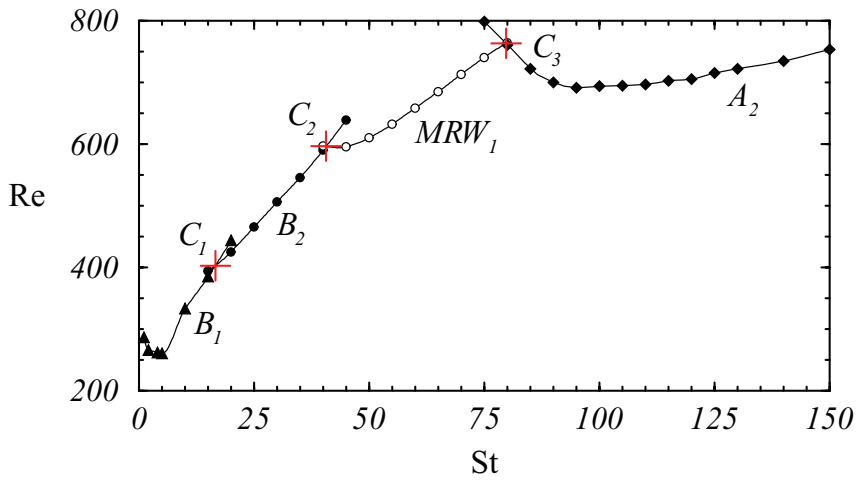

FIG. 2. (Color online) Critical Reynolds number, $\operatorname{Re}_{c}$, as a function of the Stokes number, St, for the transition from the basic state to the different three-dimensional states, $B_{1}, B_{2}, \mathrm{MRW}_{1}$, and $A_{2}$. The crosses correspond to the three bicritical points, $C_{i}$; symbols are computed critical points, and solid lines are splines. Adapted from Ref. [19]. 
modes $(A$ and $B)$ bifurcate first, while at intermediate values, the quasiperiodic mode, in the form of modulated rotating waves (MRW), leads the process. The subscript 1 or 2 indicates the azimuthal wave number $n$ (Fourier mode) of the bifurcated solution. Curves with solid triangles, circles, and diamonds are the linear stability curves for $B_{1}, B_{2}$, and $A_{2}$ synchronous modes, while circumferences correspond to the quasiperiodic mode $\mathrm{MRW}_{1}$.

Three-dimensional synchronous states appear when a pair of real eigenvalues cross the unit circle at +1 in the complex plane. As its name indicates, the synchronous states only possess the frequency associated with the forcing and, since they are no longer axisymmetric, the symmetry $\mathrm{O}(2)$ has been broken. Nevertheless, there still remain the discrete symmetry $R_{2 \pi / n}$ (a rotation of angle $2 \pi / n$ around the axis) and its powers, and a collection of $n$ reflection planes at angles $\pi / n$ apart. The rotation and meridional reflection symmetries just described generate the so-called $\mathrm{D}_{\mathrm{n}}$ symmetry group (or dihedral group) with $2 n$ elements, consisting of $n$ rotations (including the identity) and $n$ meridional reflections. The modes $B$ are invariant under the symmetry $H$, while the $A$ modes are not. In particular, the complete symmetry group of the bifurcated mode $B_{n}$ is $\mathrm{D}_{\mathrm{n}} \times \mathrm{Z}_{2}^{\mathrm{ST}} ; \mathrm{D}_{\mathrm{n}}$ is generated by the rotation $R_{2 \pi / n}$ and the reflection $K_{\beta_{0}}$, and $\mathrm{Z}_{2}^{\mathrm{ST}}$ is produced by $H$. The angle $\beta_{0}$ characterizes the solution considered, and there is a continuous family of solutions $\left(0 \leqslant \beta_{0}<2 \pi\right)$ bifurcating simultaneously in the pitchfork of revolution.

\section{DYNAMICS CLOSE TO THE CODIMENSION-2 POINT}

The intersection of two linear stability curves determines what is called a codimension- 2 or bicritical point. For instance, in Fig. 2, there are three points of this kind (crosses), because of the $B_{1}-B_{2}, B_{2}-\mathrm{MRW}_{1}$, and $\mathrm{MRW}_{1}-A_{2}$ intersections. The present study is focused on the analysis of the first codimension-2 point, $C_{1}$, where the modes $B_{1}$ and $B_{2}$ bifurcate simultaneously, and which is located at $\left(\mathrm{St}_{c}, \mathrm{Re}_{c}\right)=$ (15.636,394.57). Comprehensive numerical explorations of the two-dimensional parameter space around this bicritical point $C_{1}$ have been performed, looking at the subsequent bifurcations of the $B_{1}$ and $B_{2}$ states, their interactions and multiplicity, and transitions between them. A summary of these results is detailed in Fig. 3, which shows the critical Reynolds number, $\operatorname{Re}_{c}$, as a function of the Stokes number, $\mathrm{St}$, near $C_{1}$. The solid black curves of this figure are the same bifurcation curves that appear in Fig. 2, but without symbols, which are used for the bifurcated states. For instance, on the left side of $C_{1}, B_{1}$ modes (black circles) bifurcate first, while on the other side $B_{2}$ modes (blue solid triangles) do. In addition to these known solutions, other states that might stem from $C_{1}$ are achieved. These novel states are the rotating waves $B_{1}$ RW (blue circumferences) that bifurcate from $B_{1}$, the $B_{1}-B_{2}$ mixed modes (violet solid squares) that bifurcate from $B_{2}$, and the bursting solutions (red solid diamonds) covering a large region in parameter space. In the following sections, all of these states are discussed in detail. Some additional curves are sketched to delimit their regions of existence. The inset in Fig. 3 displays a zoom near the linear stability curve at $\mathrm{St}=16$ in order to unravel the different states that appear very close to the $B_{2}$ bifurcation curve. Similar cascades of bifurcations

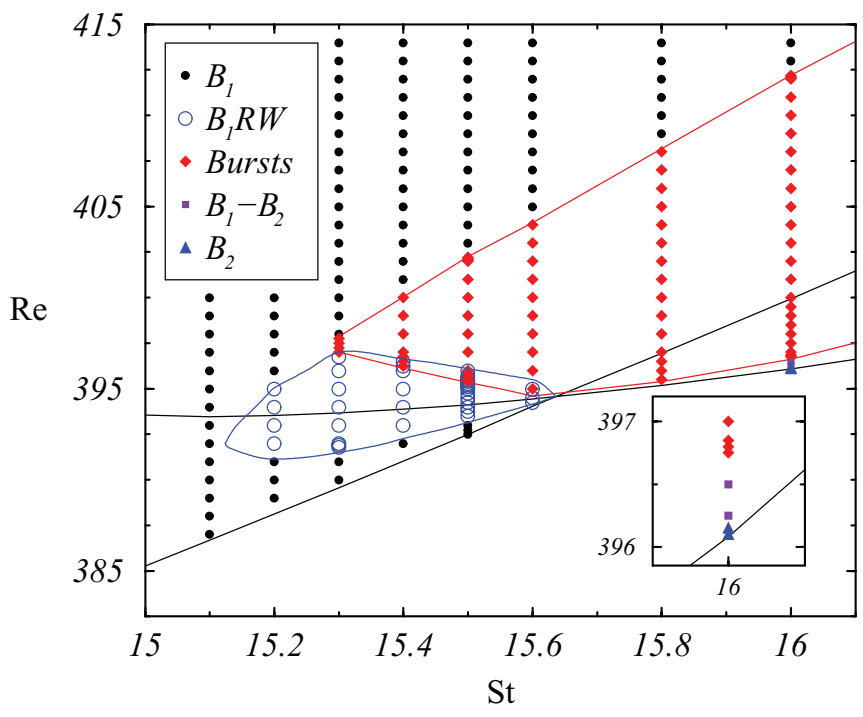

FIG. 3. (Color online) Families of bifurcated states near the bicritical point $C_{1}$. The bifurcation curves from the base state are indicated as black solid lines.

resulting in flows with complex dynamics have been reported in an annulus of radius ratio close to one also driven by axial oscillations of the sidewall [8]. In this problem, the azimuthal wave numbers of the bifurcated solutions are at least one order of magnitude larger than in our problem, so there is a strong competition between the bifurcating modes. Consequently, the system suffers a rapid succession of bifurcations in a very narrow parameter range and cannot be resolved numerically. In our scenario, this succession of bifurcations is discernible because of the relatively small wave numbers, as is exhibited in the inset of Fig. 3.

\section{A. Below the bicritical point}

As can be observed in Fig. 3, for Stokes number values below $C_{1}$, St $<\mathrm{St}_{c}=15.636$, the bifurcated mode $B_{1}$ exists for a wide range of Reynolds numbers. However, on the vicinity of the codimension- 2 point, this mode becomes unstable in a small Re region before becoming stable again. The synchronous mode $B_{1}$ preserves the $H$ symmetry. Regarding to the spatial symmetries, $\mathrm{O}(2)$ reduces to $\mathrm{D}_{1}$, generated by the reflection symmetry about a meridional plane $K_{\beta_{0}}$, and no rotational symmetry remains; the notation $Z_{2}$ is also used in the literature instead of $D_{1}$. Therefore, the symmetry group of $B_{1}$ is $\mathrm{D}_{1} \times \mathrm{Z}_{2}^{\mathrm{ST}}$.

\section{1. $B_{1} R W$ solution}

Starting with a pure $B_{1}$ state and increasing Re for frequencies within the range $15.1<\mathrm{St}<\mathrm{St}_{c}=15.636$, the mode $B_{1}$ undergoes a bifurcation, becoming a modulated rotating wave $B_{1}$ RW (blue circumferences in Fig. 3). Figure 4(a) depicts the time series of the axial velocity of one of these $B_{1}$ RW states in a fixed point near the sidewall at midheight $(r=0.9, z=\theta=0)$ that looks periodic and very similar to the corresponding time series for $B_{1}$. However, by looking at the maximum values of this time series over a long time interval, a slow variation with a large period is clearly observable in Fig. 4(b); 
(a)

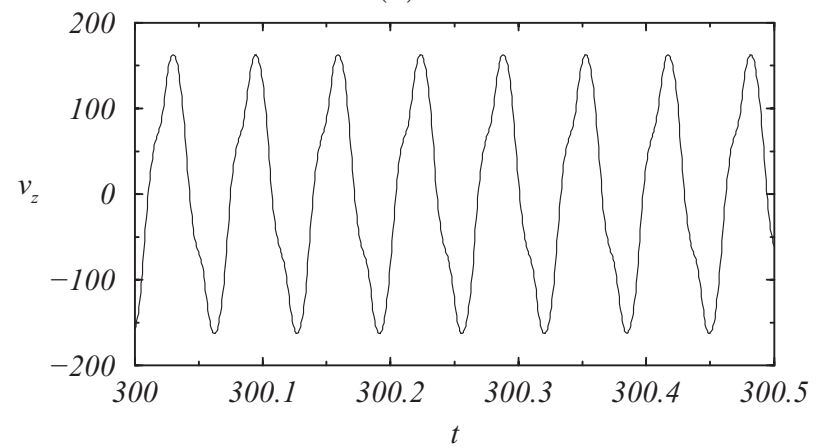

(b)

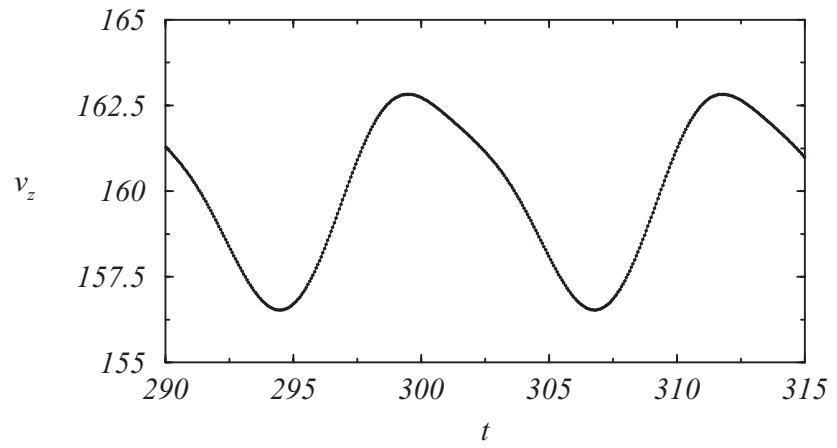

FIG. 4. Rotating-wave solution $B_{1} \mathrm{RW}$ for $(\mathrm{St}, \mathrm{Re})=(15.5,395)$. Time series of the near-wall axial velocity at midheight $(r=0.9, z=$ $\theta=0$ ). Panel (a) shows about eight forcing periods. Panel (b) plots the maximum values in (a), which displays the slow variation of the axial veocity due to the slow precession rate of the rotating wave, in a much larger time scale.

for the depicted solution at $(\mathrm{St}, \mathrm{Re})=(15.5,395)$, its value is $T_{2} \approx 12.321 \approx 190.98 T_{1}$, two orders of magnitude larger than the forcing period $T_{1}$. Figures 5(a) and 5(b) compare the common mode $B_{1}$ with the $B_{1} \mathrm{RW}$, and, by looking at the latter after 28 forcing periods in Fig. 5(c), its rotating nature becomes evident; the solution has rotated about $53^{\circ}$, in full agreement with the measured precession frequency $f_{2}=1 / T_{2} \approx 0.0812$. In fact, this state is a modulated rotating wave, because the flow structure changes during the forcing period; nevertheless, if the flow is strobed with the forcing frequency, the structure looks the same but rotated a certain angle, as shown in Figs. 5(b) and 5(c).

$B_{1} \mathrm{RW}$ is a quasiperiodic solution with two well-defined frequencies, the forcing frequency $f_{1}=\mathrm{St}=15.5$ and a much smaller frequency related with the rotation, $f_{2}=0.0812$. Figure 6(a) exhibits the power spectral density (PSD) of Fig. 4(b), showing a single frequency $f_{1}$ and its first harmonic; the very small second frequency $f_{2}$ is made apparent at the inset showing $f_{1} \pm j f_{2}$ for $j=1,2$, and 3 , in the form of additional peaks very close to the $f_{1}=15.5$ peak. In order to understand the reason of such small frequencies, Fig. 6(b) exhibits the periods $T_{2}$ for the $B_{1} \mathrm{RW}$ states in the region where these solutions exist. It is observed that the period goes to infinity, so the precession frequency $f_{2}=1 / T_{2}$ goes to zero along the bifurcation curve $B_{1} \rightarrow B_{1} \mathrm{RW}$. The pure mode $B_{1}$ does not precess, because it is $K_{\beta}$-reflection invariant, and there is a continuous family of $B_{1}$, the group orbit of $\mathrm{SO}(2)$ acting on them. What occurs is that in the bifurcation $B_{1} \rightarrow$ $B_{1} \mathrm{RW}$, the $K_{\beta}$-reflection symmetry is broken (as it is discussed shortly), and the bifurcated solutions start to drift along the group orbit, resulting in a (modulated) rotating wave. Although the bifurcated state is quasiperiodic (it has two frequencies, so it lives on a two-torus), the bifurcating eigenvalue is real, and the second frequency comes from the symmetry breaking and the corresponding drift along the group orbit [21]; the bifurcation is a pitchfork breaking $K_{\beta}$, that generates a drift because of the continuous family of $B_{1}$ solutions. It has been manifested in Ref. [21] that the period $T_{2}$ must vary as the inverse of the square root of the distance to the bifurcation point, a result consistent with the periods computed and shown in Fig. 6(b). The curves displayed in the figure are splines to guide the eye, except the curves for $\mathrm{St}=15.2$ that correspond to square-root fits. The data adjust very well, corroborating the mentioned square-root law. The largest period computed (a)

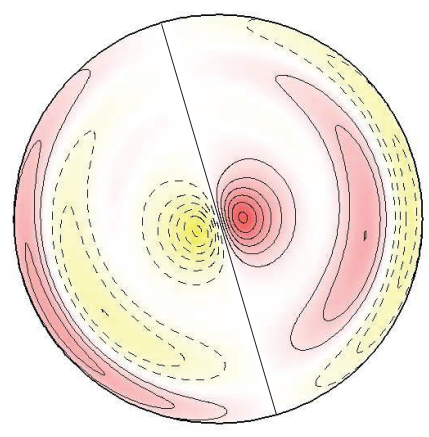

$B_{1}$ (b)

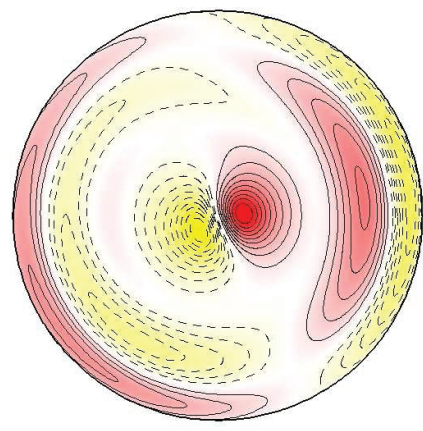

$B_{1} R W$ at $t=t_{0}$ (c)

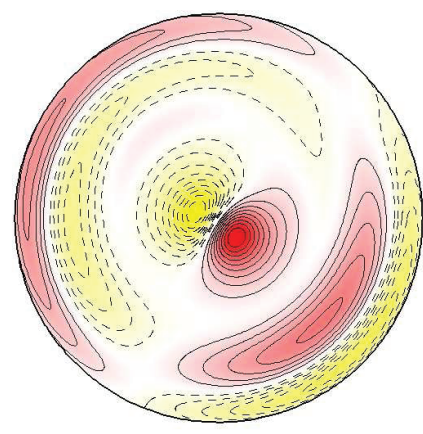

$B_{1} R W$ at $t=t_{0}+28 T_{1}$

FIG. 5. (Color online) (a) Pure $B_{1}$ mode at $(\mathrm{St}, \mathrm{Re})=(15.5,393)$. The straight line is the $K_{\beta}$ reflection symmetry axis. [(b) and (c)] Rotating-wave solution $B_{1} \mathrm{RW}$ for $(\mathrm{St}, \mathrm{Re})=(15.5,395)$. All snapshots are taken at the beginning of a forcing period; (b) and (c) are taken 28 forcing periods apart. Plots show axial vorticity contours at the horizontal section $z=-\Gamma / 4$. Solid (dashed) contours are positive (negative); light and dark (yellow and red online) colors correspond to negative and positive values. This contour-color convention is used in all subsequent cross sections. 
(a)

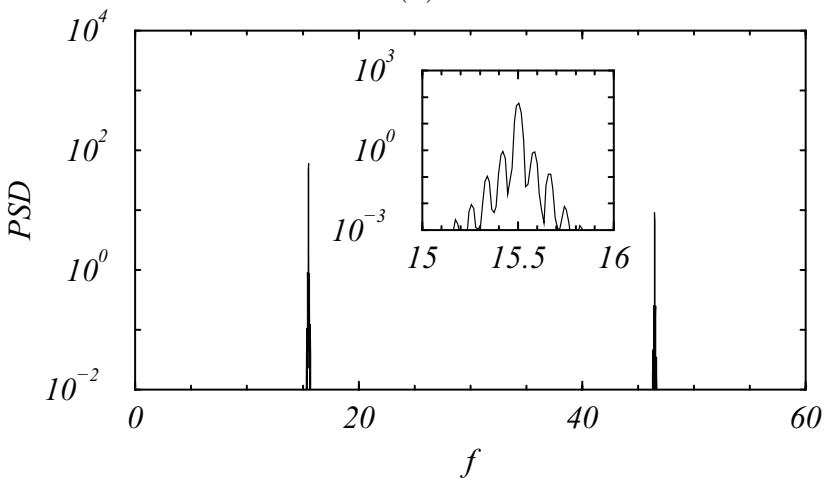

(b)

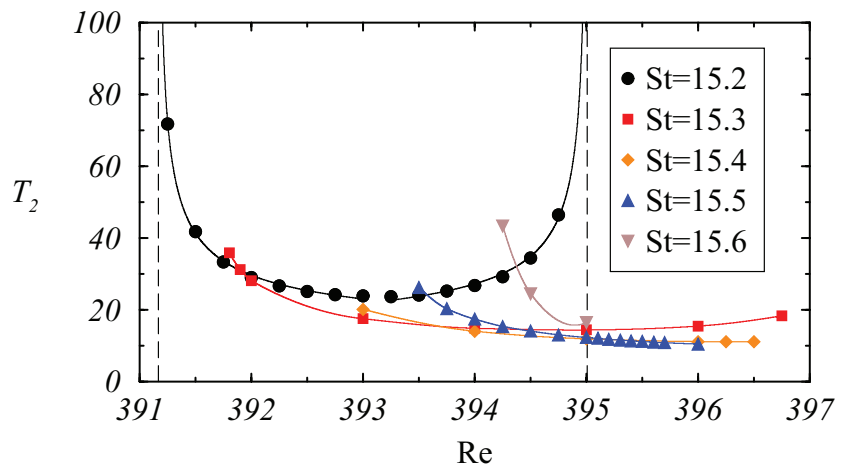

FIG. 6. (Color online) (a) FFT of the time series in Fig. 4(b). (b) Period $T_{2}$ of the $B_{1}$ RW solutions as a function of Re for different Stokes numbers St as indicated.

corresponds to the curve for $(\mathrm{St}, \mathrm{Re})=(15.2,395)$, which gives $T_{2} \approx 259$, and which in the figure is out of range.

Concerning the symmetries of the $B_{1}$ RW solutions, it is obvious from Figs. 5(b) and 5(c) that the reflection symmetry $K_{\beta}$ has been broken, so no purely spatial symmetry remains. This can be verified by computing the symmetry parameter of this $B_{1}$ RW solution, which gives $S_{K_{\beta}}\left(B_{1} \mathrm{RW}\right)=0.478$, to be compared with the value corresponding to the symmetric solution $B_{1}$ in Fig. $5(\mathrm{a}), S_{K_{\beta}}\left(B_{1}\right)=8.29 \times 10^{-10}$. This parameter is an appropriate measure of the symmetry breaking, and it is plotted in Fig. 25(a) in order to be contrasted with other solutions. Figure 7 displays the contributions of the first [Fig. 7(a)] and second [Fig. 7(b)] azimuthal Fourier components of the $B_{1}$ RW solution and helps in understanding the reason behind the breaking of the $K_{\beta}$ symmetry. First, the angle $\beta_{2}$ for the azimuthal Fourier component $n=2$ is not the same in different points of the domain. Figure 7(b) shows that close to the sidewall and in the bulk the flow possesses a different symmetry axis (straight lines in the figure), and they differ in about $6^{\circ}$. Moreover, in both modes the $\beta$ angles of the $K_{\beta}$ symmetry do not satisfy the relation $\beta_{2}=2 \beta_{1}$ : From the figure, $\beta_{1} \approx 55^{\circ}$ and $\beta_{2} \approx 168^{\circ}$ (the averaged value). Therefore, in the expression of the symmetry parameter (9), both values $\sin ^{2}\left(\beta_{2}-2 \beta_{1}\right)$ and $\sigma_{\beta_{2}}^{2}$ differ from zero. (a)

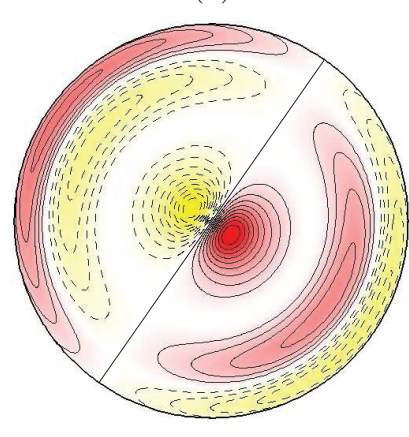

$n=1$ (b)

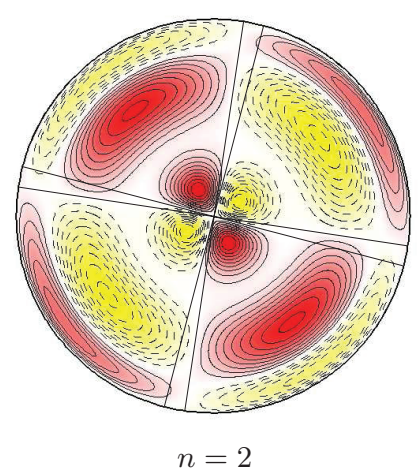

FIG. 7. (Color online) Axial vorticity contours of the rotatingwave solution $B_{1}$ RW in Fig. 5(c). The plots show the contributions of the first $(n=1)$ and second $(n=2)$ Fourier modes. Straight lines are reflection $K_{\beta}$ symmetry axes. Contour-color convention as in Fig. 5.
About the spatiotemporal symmetry $H$, since the solution is quasiperiodic and synchronous with the forcing, it cannot be considered $H$ symmetric. However, the Fourier transform of the axial velocity on the midplane shown in Fig. 6(a) has zero (or very small) even temporal Fourier components, indicating that the solution is almost $H$ symmetric. There are two reasons for this approximate symmetry. The first one is that the amplitude of the modulation due to the second frequency is very small; comparing Figs. 4(a) and 4(b), it is perceived that the modulation amplitude is about $1 \%$ of the amplitude of the axial velocity. The second reason is that the two frequencies are almost in resonance, so $T_{2}$ is very close to an integer multiple of $T_{1}: T_{2}=190.98 T_{1} \approx 191 T_{1}$. When the ratio is integer, the solution is periodic and exactly satisfies the $H$ symmetry (with period $T_{2}$ ).

\section{Bursting solution}

For forcing frequencies close to the codimension-2 point $C_{1}, 15.3 \leqslant \mathrm{St}<\mathrm{St}_{c}=15.636$, the stable $B_{1}$ existing at large Re does not bifurcate into the rotating-wave solution $B_{1} \mathrm{RW}$ when decreasing Re but undergoes a bifurcation to a more complicated bursting state (red solid diamonds in Fig. 3). The bursting state and the $B_{1} \mathrm{RW}$ coexist in a narrow hysteretic region observable in Fig. 3 and become unstable exiting the region, one evolving into the other.

Figure 8(a) exhibits the time series of the energies of the first and second azimuthal Fourier components of the bursting solution at $(\mathrm{St}, \mathrm{Re})=(15.5,400)$. Both modes have comparable energies, and the resulting state alternates between both, showing plateaus where the energy is almost constant followed by rapid excursions, thus the name bursting solution. These solutions are periodic in time, and the period from the energy plot is $T_{2} \approx 8.2417$. The solution is most of the time very close to a $B_{2}$ mode, with excursions to a mode $B_{1}$. But the energy, being a global measure, does not capture the details of the flow. Figure 8(b) provides the evolution of the maximum values of the axial velocity at $z=0$ near the wall. Measuring the period using this figure, a different result is obtained, $T_{2} \approx 33.065$. This value is 4 times the one obtained from the energy plot. The reason behind this is the fact that the energy does not distinguish among solutions with the same structure but only those rotated at a certain angle. 
(a)

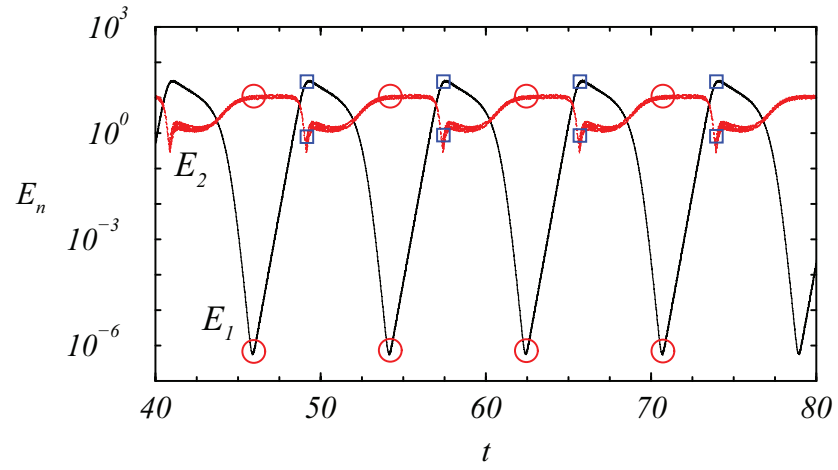

(b)

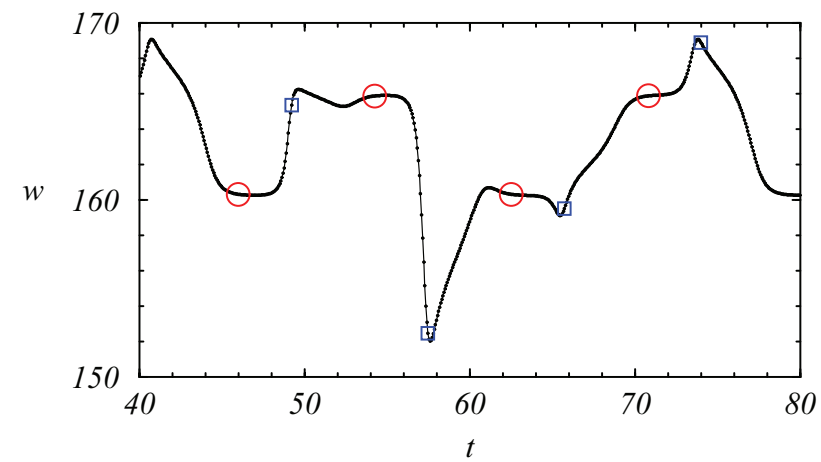

FIG. 8. (Color online) Bursting solution at $(\mathrm{St}, \mathrm{Re})=(15.5,400)$. Time series of (a) the energy of the Fourier modes $E_{1}$ and $E_{2}$, and (b) maximum values of the near-wall axial velocity at midheight $(r=0.9, z=\theta=0)$ [same plot as in Fig. 4(b)].

In Fig. 9, contours of axial vorticity at $z=-\Gamma / 4$ corresponding to consecutive minima of $E_{1}$ and $E_{2}$ in Fig. 8(a) are depicted; $t_{0}$ is taken at the beginning of the forcing period. The solutions are almost identical to the pure synchronous modes $B_{1}$ (at the minimum of the $n=2$ mode, blue open squares in the figures) and $B_{2}$ (at the minimum of the $n=1$ mode, red circumferences in the figures). But the solutions at two consecutive minima of the $n=2$ mode (almost pure $B_{1}$ modes) are rotated $\pi / 2$; therefore, the initial state is recovered after four alternations of the synchronous modes and the discrepancy between the measures of $T_{2}$ in the previous paragraph gets explained.

As it happens with the rotating waves, the bursting solution is also quasiperiodic and there are two well-defined frequencies, the forcing frequency, $f_{1}=\mathrm{St}=15.5$, and a very small frequency associated with the bursts, $f_{2}=0.0302$. Figure 10(a) shows the Poincare section of the bursting solution considered, using two axial velocities measured at $z=0$ and $z=0.95$, close to the sidewall. The Poincaré section is quite convoluted, and the slowdown where the solution is close to a pure $B_{2}$ mode and the fast excursion approaching a pure $B_{1}$ mode are captured. The bullets represent 513 consecutive points in the reduced space and corroborate that the mentioned $f_{2}$ is correct, because $f_{1} / f_{2}=512.51$, and a whole lap around the cycle is done. The blue open squares and red circumferences correspond to the minima of the first and second Fourier mode displayed in Fig. 9. Moreover, there seem to be two accumulation points, which are the two possibilities
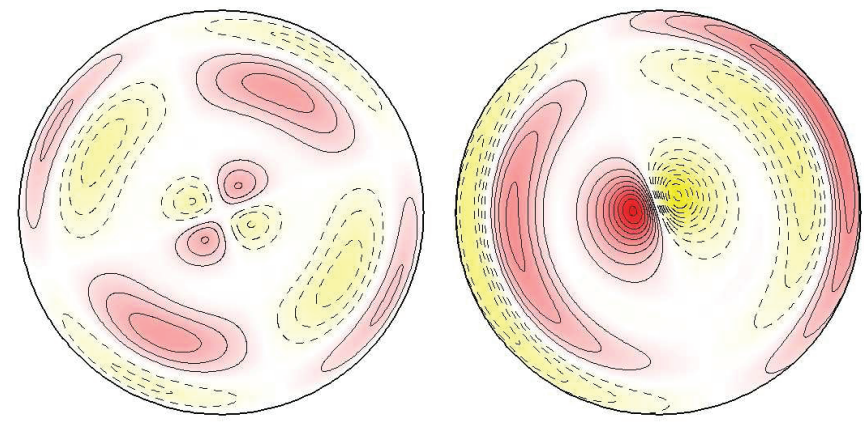

$t=t_{0}$

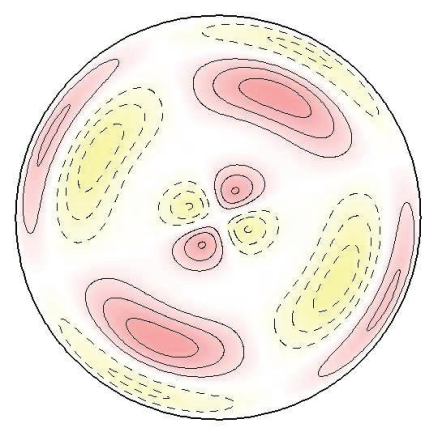

$t=t_{0}+256 T_{1}$

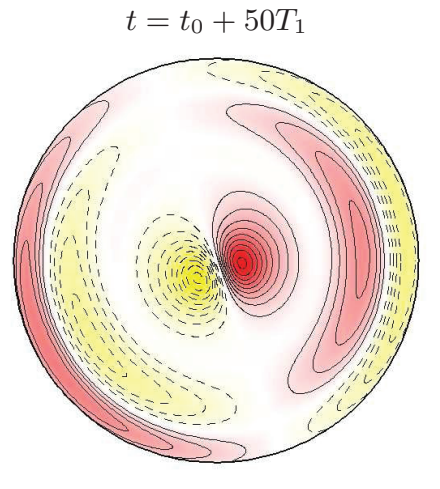

$t=t_{0}+306 T_{1}$
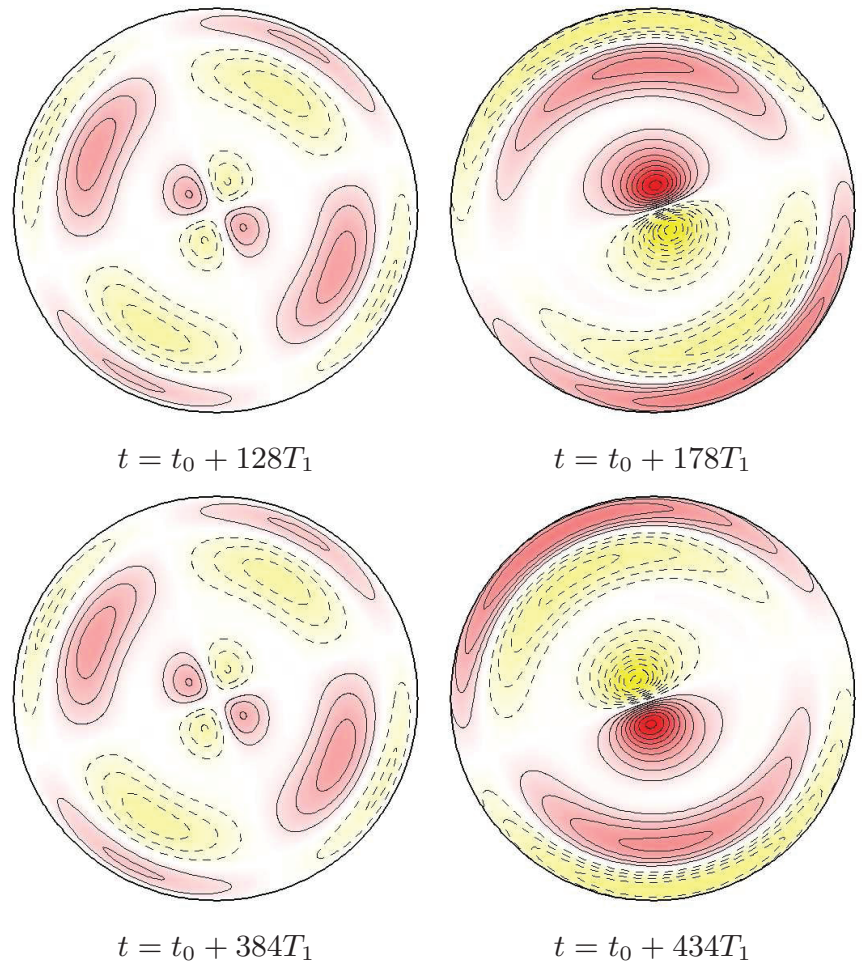

FIG. 9. (Color online) Axial vorticity contours at $z=-\Gamma / 4$ and different times of the bursts at $(\operatorname{St}, \operatorname{Re})=(15.5,400) ; t_{0} \approx 45.94$ is at the beginning of the forcing period and each snapshot corresponds to consecutive minima of $E_{1}$ and $E_{2}$. Contour-color convention as in Fig. 5 . 
(a)

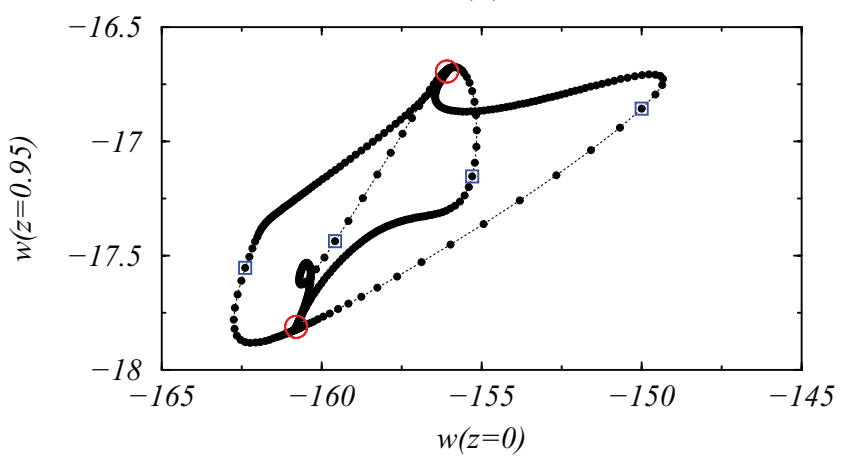

(b)

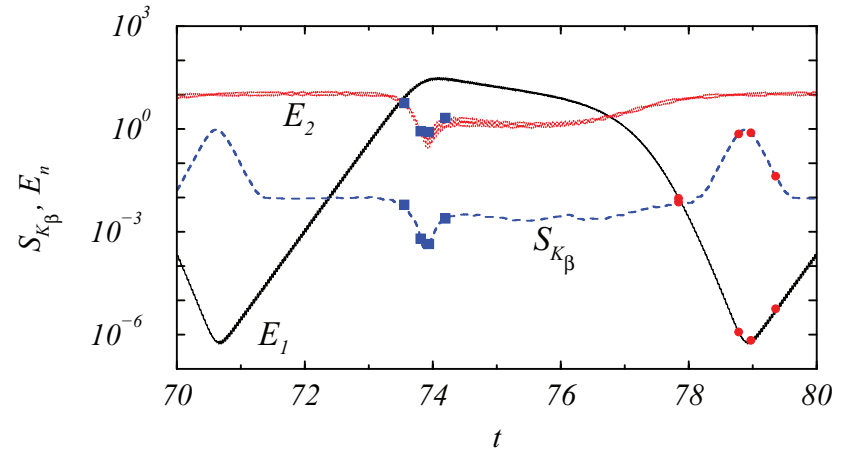

FIG. 10. (Color online) Bursting solution at $(\mathrm{St}, \mathrm{Re})=(15 \cdot 5,400)$. (a) Poincaré section (bullets) using two axial velocities measured near the wall $\left(r=0.9, \theta=0\right.$, and $z$ as indicated). The minima of $E_{1}$ (circumferences) and $E_{2}$ (open squares) indicated in Figs. 8 and 9 are also plotted. (b) Time evolution of the $K_{\beta}$ asymmetry parameter, $S_{K_{\beta}}$; symbols refer to Fig. 11.

for the $B_{2}$ modes depicted in Fig. 9. Apparently, there are two ways of entering and another two of escaping each one of these accumulation points (pure $B_{2}$ modes).

This solution seems to preserve the spatial symmetries of the pure modes, because most of the time is close to a pure synchronous mode, but it is not the case. Figure 10(b) exhibits the evolution of $S_{K_{\beta}}$ along a burst: This parameter is most of the time constant, except for a remarkable peak and valley that coincide with the minima of the energies, also plotted in the figure. Therefore, the reflection symmetry is broken mainly when the energy of the $n=1$ mode is minimum. Figure 11 helps to understand how this symmetry-breaking process takes place. The first row shows a time sequence of the first Fourier mode near the peak of $S_{K_{\beta}}$ (minimum of the first mode, red circles in the figure), while the second one exhibits the behavior of the second mode at the valley (minimum of the second mode, blue solid squares in the figure). At the peak, the first mode becomes almost negligible (the amplitude decreases three orders of magnitude and the energy seven), while precessing in a fashion similar to the $B_{1} \mathrm{RW}$ of the former subsection; the total rotation of the first Fourier mode is exactly $\pi / 2$. When this mode is rotating, the reflection planes between the first and the second Fourier mode become uncorrelated, thus enhancing $S_{K_{\beta}}$. The second mode does not rotate continuously at any stage, and it changes sign when it is close to the minimum of the second mode; the change of sign
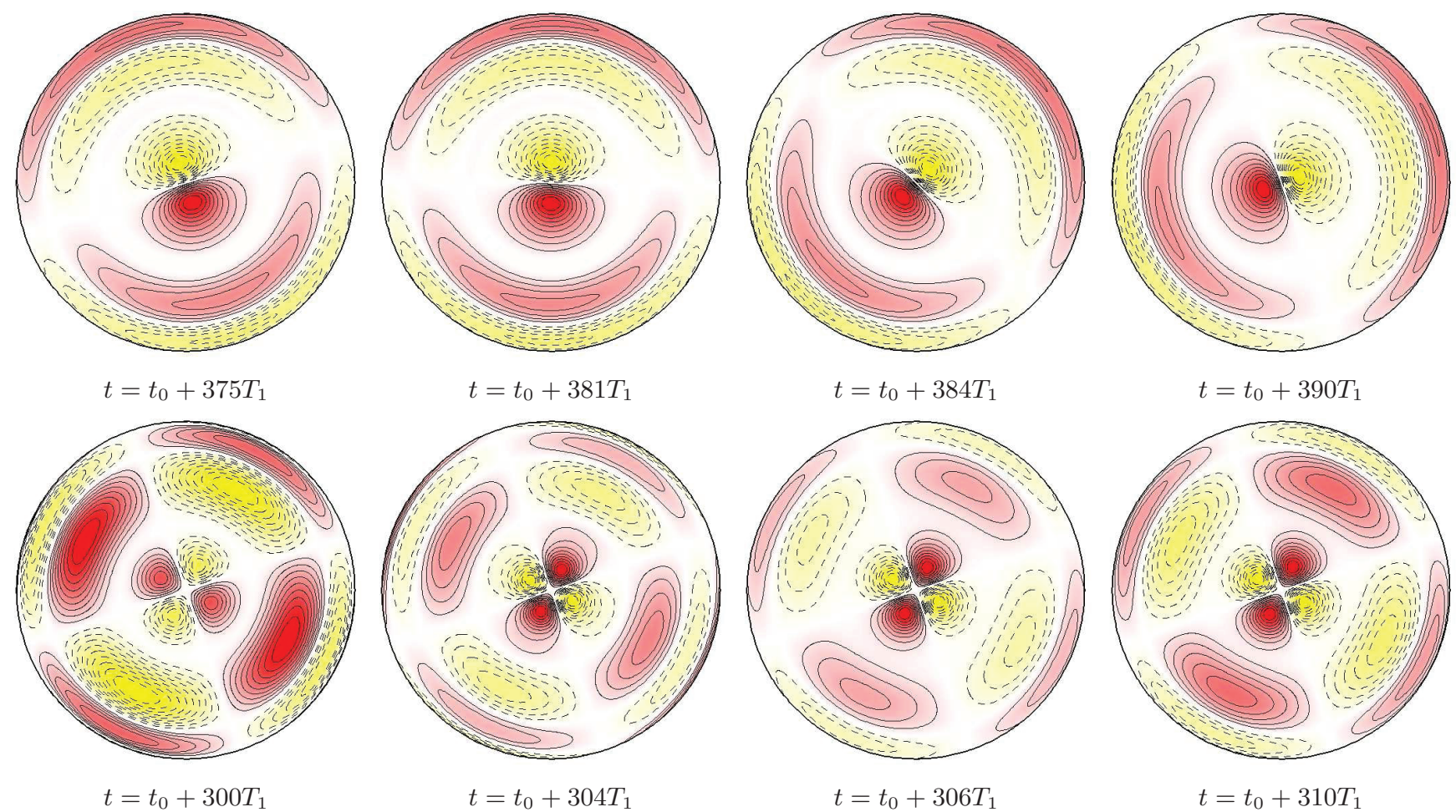

FIG. 11. (Color online) Axial vorticity contours at $z=-\Gamma / 4$ and different times of the bursts at $(\mathrm{St}, \mathrm{Re})=(15.5,400) ; t_{0}$ is at the beginning of the forcing period. The first row represents the first Fourier mode contribution near its minimum, while the second one the analogous for the second mode. The snapshots shown correspond to symbols in Fig. 10(b). Contour-color convention as in Fig. 5. 
(a)

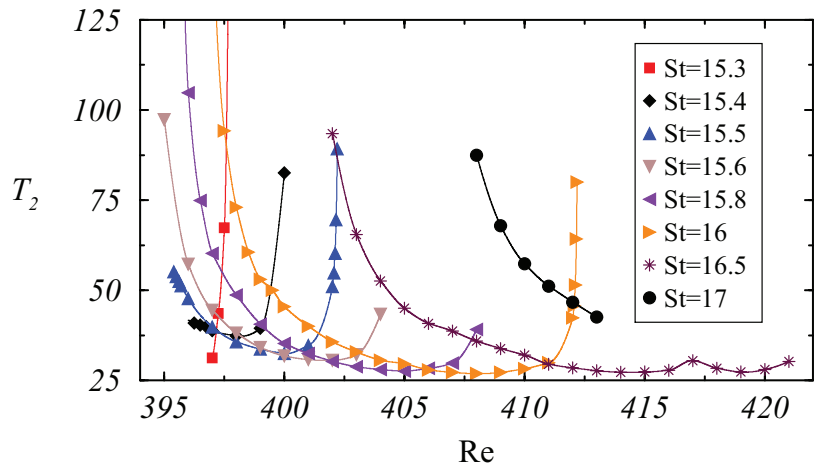

(b)

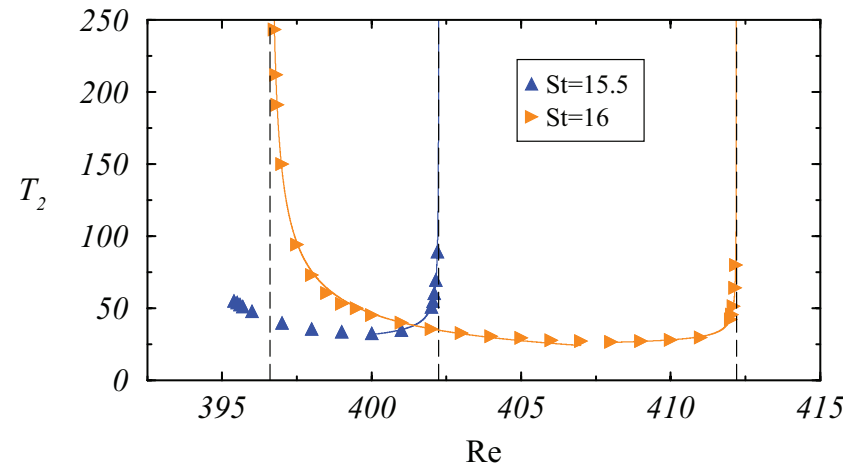

FIG. 12. (Color online) (a) Period $T_{2}$ of the bursting solutions. The symbols are computed values, and the solid lines are splines plotted to guide the eye. (b) Square-root fits to some of the SNIC bifurcations in (a). The dashed lines indicate the Re bifurcation value obtained from the fit.

is not uniform but begins near the axis and propagates towards the sidewall. Therefore, the valley in $S_{K_{\beta}}$ is created when the second mode becomes minimum. The maximum of $S_{K_{\beta}}$ is a good measure of the symmetry breaking for this solution, and it is plotted in Fig. 25 for comparison with other solutions.

Regarding the spatiotemporal $H$ symmetry, we note that it is not preserved because of the second frequency associated with the bursts, which makes it a quasiperiodic solution. Curiously, but for the same reasons as the rotating-wave solution $B_{1}$ RW, the even temporal Fourier components of the axial velocity on the midplane are very close to zero, indicating that the solution is almost $H$ symmetric, as shown in Fig. 23(b).

In order to understand the origin of the small frequency of the bursting solutions and the kind of bifurcations they undergo, the period $T_{2}$ is plotted in Fig. 12(a) as a function of the Reynolds number $\mathrm{Re}$ for different $\mathrm{St}$ values. The periods $T_{2}$ become very large when approaching the upper Re bifurcation curve in Fig. 3, where $B_{1}$ bifurcates to the bursting solution. This happens not only for $\mathrm{St}<\mathrm{St}_{c}$, below the codimension- 2 point $C_{1}$, but also for $\mathrm{St}_{c}<\mathrm{St} \leqslant 16$. This infinite period bifurcation is called a saddle-node infinite cycle (SNIC) bifurcation and occurs when a saddle-node bifurcation takes place on an invariant cycle [22]. Previously to the bifurcation, there exists a stable limit cycle. At the bifurcation a saddle-node appears on the limit cycle. After the bifurcation, the saddle-node splits into two fixed points, one stable and the other unstable, destroying the limit cycle in the process. In our problem, there is a quasiperiodic solution on a two-torus, the bursting solution, instead of a limit cycle, but the dynamics on a Poincaré section of the torus are the same as the dynamics just described for the SNIC bifurcation. The fixed points that appear on the SNIC bifurcation correspond in our case to the $B_{1}$ synchronous solution. This scenario is common in fluid dynamics and is described in detail, for example, in Ref. [23] in a rotating convection problem. A characteristic signature of a SNIC bifurcation is the square-root law followed by the period when approaching the bifurcation, which scales as the inverse of the square root of the distance to the bifurcation point [24]. Indeed, the periods shown in Fig. 12(a) that connect the bursts with the $B_{1}$ mode follow the square-root law, as shown in Fig. 12(b). The bifurcation of the bursting solutions for low Re values is also an infinite period bifurcation for $\mathrm{St} \in[15.8,17]$, above the bicritical $C_{1}$ point, as is clearly seen in Fig. 12(a). In this scenario, $T_{2}$ also follows the square-root law, as shown in Fig. 12(b), so it is considered a SNIC bifurcation, this time with the synchronous mixed-mode solution $B_{1}-B_{2}$ that is described in Sec. III B. However, below $C_{1}$, there are no stable limit cycles available, and the bifurcation of the bursts takes place at a finite value of the period and might be considered a saddle-node bifurcation of bursting solutions. When approaching the bicritical point $C_{1}$ from below ( $\mathrm{St}<\mathrm{St}_{c}$ ), the period at the bifurcation becomes very large $(\mathrm{St}=15.5$ and 15.6$)$, suggesting that it is likely to become infinite at the $C_{1}$ point, exactly what happens just on the other side of $C_{1}\left(\mathrm{St}>\mathrm{St}_{c}\right)$. The periods for $\mathrm{St}=16.5$ and 17 do not go to infinity because for these large St values other branches of solutions appear and the bursting solutions no longer bifurcate from $B_{1}$, as it can be observed in Fig. 14 .

In summary, the two quasiperiodic solutions obtained below the codimension-2 point break the reflection symmetry $K_{\beta}$ and are immersed in a large parameter region where the pure $B_{1}$ synchronous mode is stable. In addition, the bifurcations from $B_{1}$ to the quasiperiodic solutions ( $B_{1} \mathrm{RW}$ and bursts) are infinite period bifurcations.

\section{B. Above the bicritical point}

For $\mathrm{St}>\mathrm{St}_{c}$, at the right side of $C_{1}$ in Fig. 3, the bifurcated mode $B_{2}$ exists in a very narrow region very close to the linear stability curve, as presented in the inset of Fig. 3. This synchronous mode becomes rapidly unstable when increasing $\mathrm{Re}$, and a synchronous mixed mode emerges subsequently. This mixed-mode solution persists longer, but rapidly becomes unstable and reverts to the bursting solution already obtained in the previous section, in an infinite period bifurcation (SNIC). By further increasing Re, the pure $B_{1}$ mode is achieved as in the previous section.

The synchronous $B_{2}$ mode (blue solid triangles in Fig. 3) has been analyzed in Ref. [19]. Summing up, this solution is synchronous and possesses the symmetry group $\mathrm{D}_{2} \times \mathrm{Z}_{2}^{\mathrm{ST}}$. The spatial symmetries $\mathrm{D}_{2}$, which are illustrated in Fig. 13(a), depicting axial vorticity contours of $B_{2}$ at $(\mathrm{St}, \mathrm{Re})=(16,396.25)$, are generated by two orthogonal reflection planes, whose product is a half-turn (a rotation of $\pi$ around the cylinder 
(a)

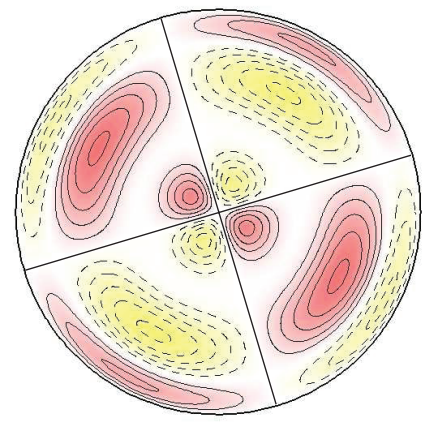

$t=t_{0}, z=-\Gamma / 4$ (b)

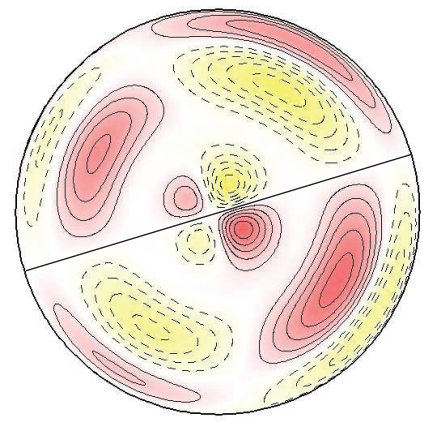

$t=t_{0}, z=-\Gamma / 4$ (c)

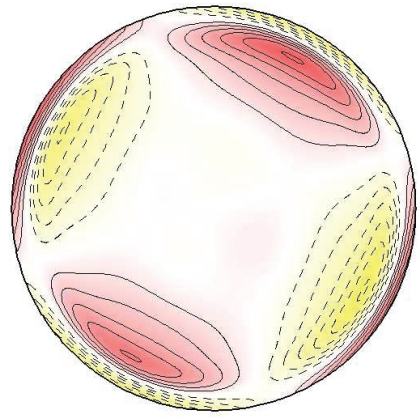

$t=t_{0}+T_{1} / 2, z=-\Gamma / 4$ (d)

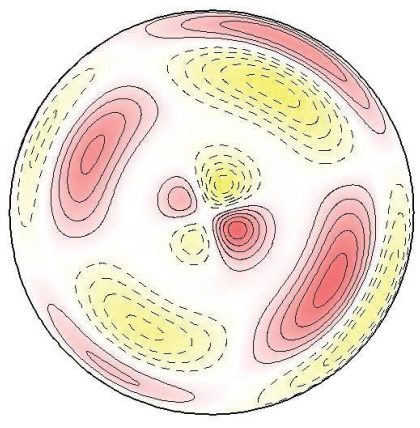

$t=t_{0}+T_{1} / 2, z=+\Gamma / 4$

FIG. 13. (Color online) (a) Axial vorticity contours of the $B_{2}$ pure mode at (St, Re) $=(16,396.15)$. [(b), (c), and (d)] Axial vorticity contours of the $B_{1}-B_{2}$ mixed mode at $(\mathrm{St}, \mathrm{Re})=(16,396.25)$, in the $z$ sections and times indicated; $t_{0}$ is at the beginning of the forcing period. The straight lines are the $K_{\beta}$ reflection symmetry axes. Contour-color convention as in Fig. 5.

axis). This pure $B_{2}$ mode is also $H$ symmetric. Since it is a pure $B_{2}$ mode, only even azimuthal Fourier components are present in this solution.

Synchronous $B_{1}-B_{2}$ mixed-mode solutions (violet solid squares in Fig. 3) emerge rapidly from the $B_{2}$ solutions. Starting from $B_{2}$ and augmenting Re, the first azimuthal Fourier mode becomes unstable, and after an oscillatory transient, a $B_{1}-B_{2}$ mixed-mode state is obtained, that is synchronous with the forcing. A steady bifurcation occurs here and in the process the $\mathrm{D}_{2}$ symmetry is lost. Figure 13(b) displays contours of axial vorticity for the mixed mode $B_{1}-B_{2}$. This solution has the same spatial symmetries as the pure $B_{1}$ mode: $\mathrm{D}_{1}$, consisting of a single reflection symmetry $K_{\beta}$. As can be observed in the figure, the even azimuthal modes are still dominant, but the presence of the modus one modifies the symmetries of the mixed solution. Figures 13(c) and 13(d) exhibit the invariance of this mixed-mode solution under the spacetime symmetry $H$. This feature is also confirmed by scrutinizing the PSD of the axial velocity on the midplane and checking the absence of the even temporal Fourier components.

The main difference between the dynamics below and above the bicritical point is the rapid sequence of bifurcations taking place above $C_{1}$, resulting almost immediately in bursting states that are quasiperiodic and possess very rich dynamics. In contrast, below $C_{1}$ the pure mode $B_{1}$ exists and is stable in a very large parameter domain, except in a region close to the codimension- 2 point, where modulated rotating waves and bursting solutions come into sight.

\section{DYNAMICS AWAY FROM THE CODIMENSION-2 POINT}

In the previous sections the dynamics and bifurcations close to the codimension- 2 point $C_{1}$ were analyzed. In the present section, the analysis to secondary bifurcations is extended in a larger region of parameter space, where a wealth of additional bifurcations is found. These new results are summarized in Fig. 14, where the different symbols correspond to numerically computed states. The previous bifurcations of Fig. 3 correspond to the region close to $C_{1}$ indicated by a dashed rectangle in Fig. 14. As in Fig. 3, the black solid curves correspond to the bifurcation curves of the basic state that meet at $C_{1}$, and the states sketched in the previous section have been included. In addition to the mentioned states, some novel states emerge away from $C_{1}$. For instance, for Stokes numbers below $\mathrm{St}_{c}$, the synchronous $B_{1}$ modes become quasiperiodic (brown open down triangles), while for $\mathrm{St}>\mathrm{St}_{c}$, new families of mixed modes (violet open squares) and bursting solutions (orange open diamonds), differing from the ones previously obtained, enter the stage. Moreover, at sufficiently high Re, chaotic solutions develop from the $B_{1} \mathrm{QP}$ and asymmetric bursts (not shown in Fig. 14). This cascade of bifurcations towards chaotic states complements the picture sketched in the previous section and helps to better understand the process described in Ref. [8].

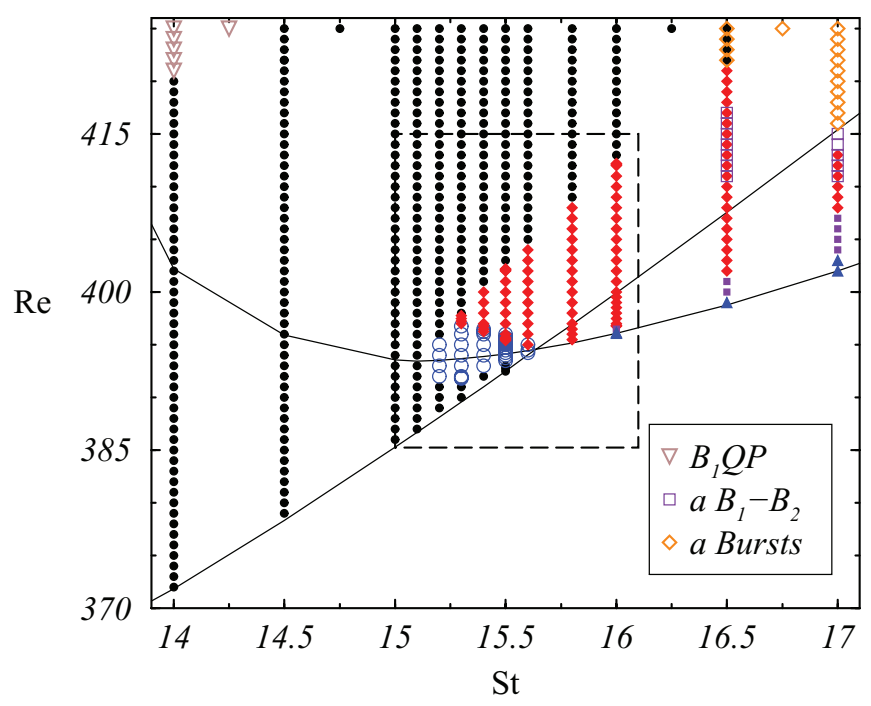

FIG. 14. (Color online) Families of bifurcated states around the bicritical point $C_{1}$. The bifurcation curves from the base state are indicated as black solid lines. The dashed rectangle corresponds to Fig. 3. The new bifurcated states away from the bicritical point $C_{1}$ are indicated in the figure legend. 
(a)

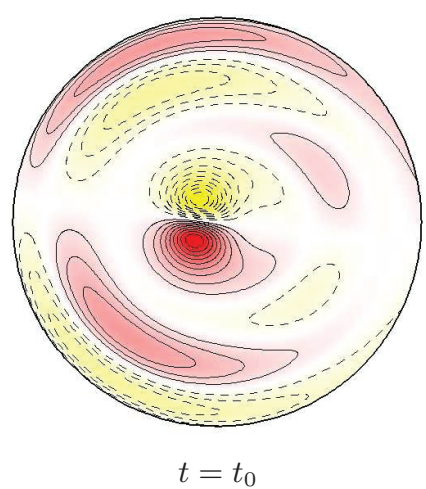

(b)

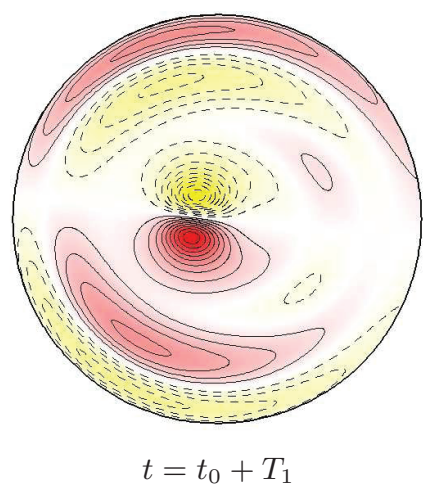

(c)

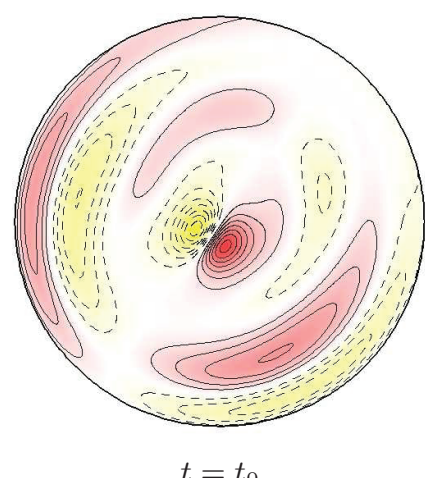

FIG. 15. (Color online) Axial vorticity contours at $z=-\Gamma / 4$ and different times; $t_{0}$ is at the beginning of the forcing period. [(a) and (b)] Quasiperiodic solution $B_{1} \mathrm{QP}$ at $(\mathrm{St}, \mathrm{Re})=(14,425)$. (c) $B_{1}$ chaotic solution for $(\mathrm{St}, \mathrm{Re})=(14,500)$; see Supplemental Material [25]. Contour-color convention as in Fig. 5.

\section{A. Small St numbers}

The pure mode $B_{1}$ is very robust for small Stokes numbers St. By increasing the Reynolds number Re enough, $B_{1}$ undergoes a sequence of bifurcations strongly resembling the Ruelle-Takens route to chaos. The periodic solution $B_{1}$ bifurcates first to a quasiperiodic solution with two frequencies, and, by further increasing Re, the torus is destroyed and a chaotic state appears. The quasiperiodic solution, $B_{1} \mathrm{QP}$ (brown open down-triangles in Fig. 14), is a $B_{1}$ mode pulsating with an additional characteristic frequency; this is clearly observed in Figs. 15(a) and 15(b), showing two axial vorticity contours at the beginning of the forcing period. They strongly resemble the pure mode $B_{1}$ in Fig. 5(a), but the intensity of the flow increases and decreases with the second frequency. By further increasing the Reynolds number, other frequencies become apparent, and eventually the $B_{1} \mathrm{QP}$ solutions acquire a chaotic pattern. A snapshot of these chaotic solutions is shown in Fig. 15(c). See Supplemental Material [25] associated with Fig. 15(c) that reproduces snapshots over 100 forcing periods taken at the beginning of the forcing period (a Poincaré section movie) and clearly exhibits the chaotic nature of this solution [25]. The main difference with $B_{1} \mathrm{QP}$ is that the reflectional symmetry $K_{\beta}$ is clearly broken. This can be verified by computing the symmetry parameter of these two solutions: $S_{K_{\beta}}\left(B_{1} \mathrm{QP}\right)=$ (a)0.0234,(b)0.0132 and $S_{K_{\beta}}$ (chaotic) $=0.362$. The two flows are no longer symmetric, but the symmetry parameter of the chaotic state is one order of magnitude larger, so the symmetry breaking can be visually observed. As both states have more than one frequency and are not periodic, the $H$ symmetry is broken too.

The chaotic character of the state at $(\mathrm{St}, \mathrm{Re})=(14,500)$ cannot be discerned in the snapshot shown in Fig. 15(c), but it is evident by comparing the time series of the energies of the first and second Fourier modes shown in Fig. 16(a) for the $B_{1} \mathrm{QP}$ and Fig. 16(b) for the chaotic state. The chaotic state is much more complex and shows random time intervals where the azimuthal mode $n=2$ is dominant. The solution alternates between pure modes, mixed modes or largely distorted modes without a precise pattern. The comparison of the Fourier transforms of the axial velocity on the midplane for the two states is shown in Fig. 17. The PSD of $B_{1} \mathrm{QP}$ is clearly quasiperiodic with two well-defined frequencies and their linear combinations, while the PSD of the chaotic state exhibits a broad band of frequencies, and only a few multiples of the forcing frequency are outstanding. Notice that in both Fourier transforms the even multiples of the forcing frequency are very (a)

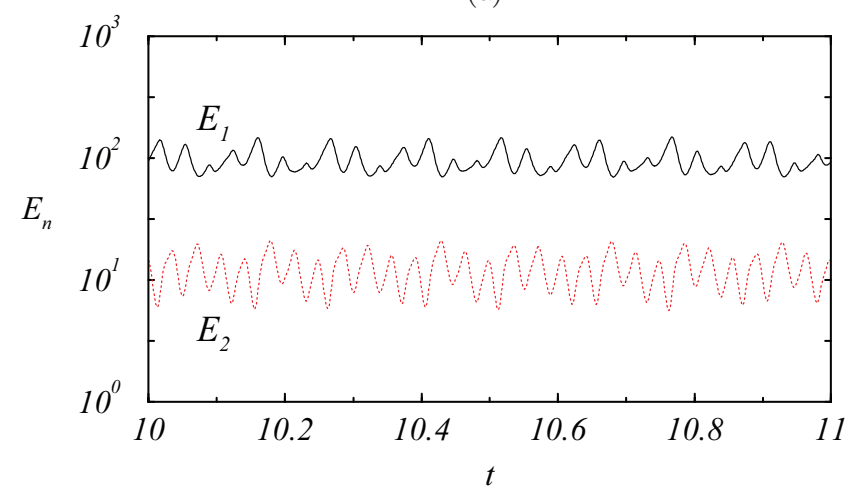

(b)

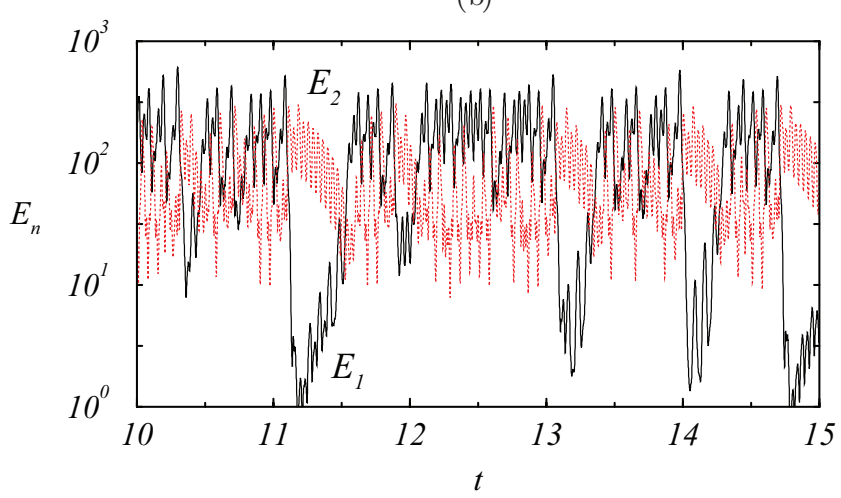

FIG. 16. (Color online) Time series of energy of the Fourier mode $E_{1}$ and $E_{2}$ for (a) the $B_{1} \mathrm{QP}$ at $(\mathrm{St}, \mathrm{Re})=(14,425)$ and for $(\mathrm{b})$ the $B_{1}$ chaotic solution at $(\mathrm{St}, \mathrm{Re})=(14,500)$. 
(a)

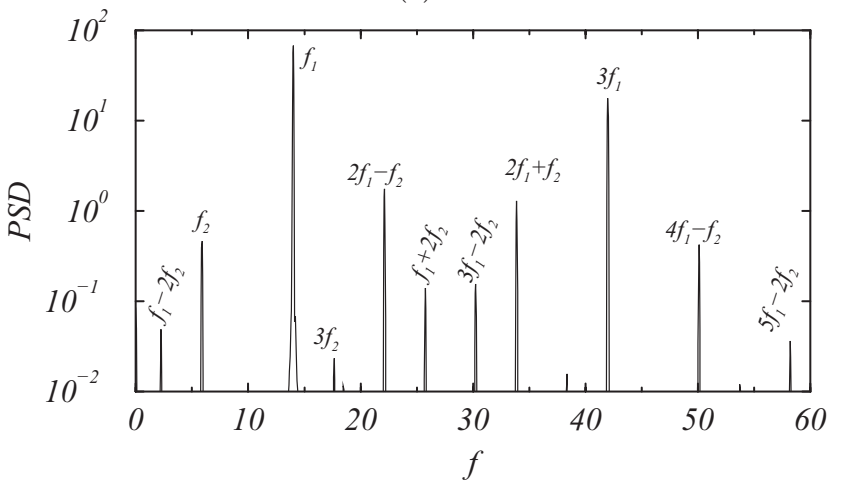

(b)

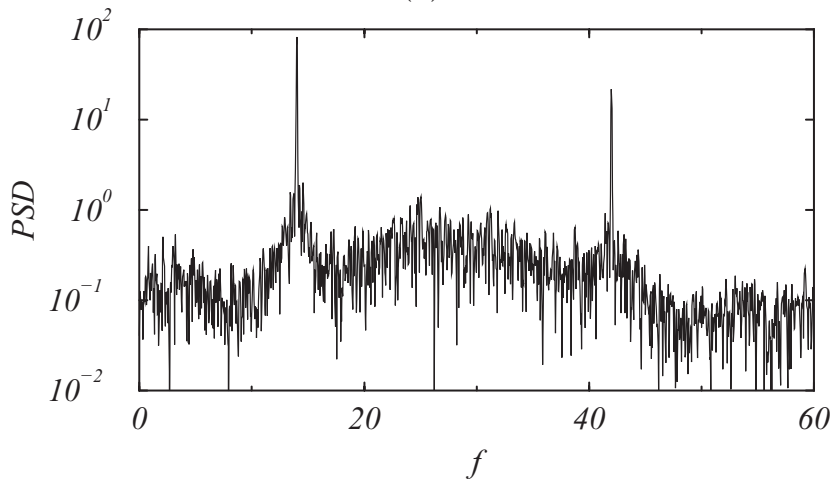

FIG. 17. FFT of the time series of the near-wall axial velocity at midheight $(r=0.9, z=\theta=0)$ for the (a) $B_{1} \mathrm{QP}$ at $(\mathrm{St}, \mathrm{Re})=(14,425)$ and (b) $B_{1}$ chaotic solution at $(\mathrm{St}, \mathrm{Re})=(14,500)$.

small, indicating that the spatiotemporal symmetry $H$ is still approximately fulfilled.

Figure 18 represents the Poincaré section of two axial velocities measured at $z=0$ and $z=0.95$ near the sidewall after performing a sampling of points every forcing period (bullets). The Poincaré section for the $B_{1} \mathrm{QP}$ displayed in Fig. 18(a) produces a perfect cycle, so this solution lives in a two-torus. The big circumferences represent four consecutive points in the reduced space, and it is observed that the second iteration approaches the initial point and the third one surpasses it, in excellent agreement with the second frequency $f_{2}=5.89$ measured from the Fourier transform in Fig. 17(a), which provides $f_{1} / f_{2}=2.38$, indicating that going around the circle takes 2.38 iterates. Figure 18(b) represents the Poincaré section of the chaotic state at $(\mathrm{St}, \mathrm{Re})=(14,500)$. The result cannot be considered a limit cycle in any case. The two-torus has been destroyed, and the points observed might be considered the Poincaré section of a chaotic attractor.

\section{B. Large St numbers}

Observing Fig. 14, for higher St than the bicritical value, two new branches of solutions turn up. These two new solutions are called asymmetric mixed modes and asymmetric bursts. The asymmetric mixed modes are very similar in some aspects to the former mixed modes but do not preserve the spatiotemporal symmetry $H$, thus the asymmetric adjective.

(a)

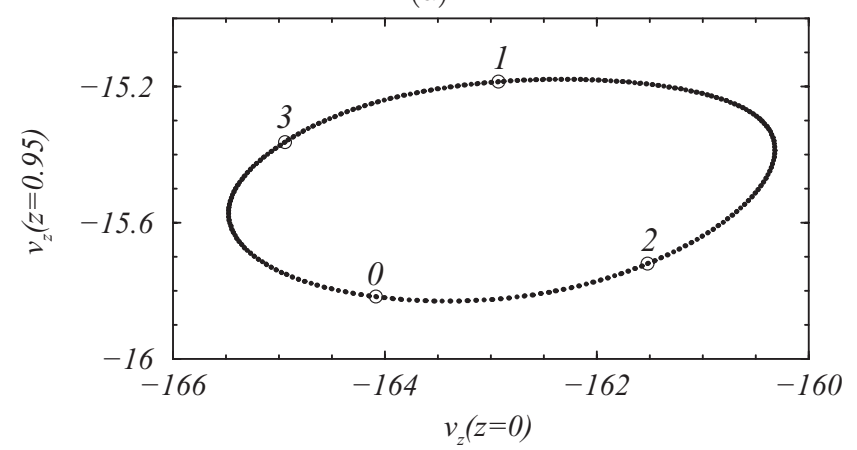

Since the asymmetric bursts bifurcate from the asymmetric mixed modes, they are called asymmetric, too. By further increasing Re above the range of Fig. 14, irregular or chaotic bursting solutions are obtained.

\section{1. $B_{1}-B_{2}$ asymmetric mixed-mode solution}

The mentioned asymmetric $B_{1}-B_{2}$ mixed-mode solutions (violet open squares in Fig. 14) are obtained far away from $C_{1}$ and its branch coexists in the parameter space with the bursts. By increasing $\mathrm{Re}$ at $\mathrm{St}=17$, the symmetric bursts of the former section become unstable, and after a transient the new asymmetric mixed mode is achieved. When continued to smaller Re, this new branch of solutions coexists with the burst branch and there is a noticeable hysteresis region, as can be seen in Fig. 14. This asymmetric mixed mode has a single characteristic time, the forcing period, and, hence, this solution is synchronous, like the $B_{1}-B_{2} H$-symmetric mixed modes discussed in Sec. III B.

In Figs. 19(a) and 19(b), contours of axial vorticity are depicted in the separated half-forcing period, and in symmetric planes with respect to $z=0$, for the asymmetric mixed mode at $(\mathrm{St}, \mathrm{Re})=(17,414)$. At first sight, the solution resembles the pure mode $B_{2}$ of Fig. 13(a). Nevertheless, the difference in strength of the four quadrants in Figs. 19(a) and 19(b) breaks the rotational symmetry of $\pi$. Therefore, this solution preserves exactly the same spatial symmetries as the pure $B_{1}$ modes do: of the continuous family of symmetries $\mathrm{O}(2)$ of

(b)

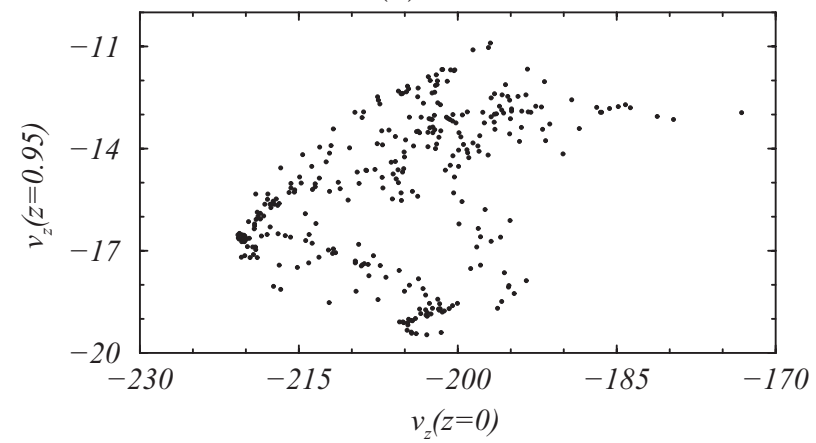

FIG. 18. Poincaré sections (bullets) using two axial velocities measured near the wall ( $r=0.9, \theta=0$ and $z$ as indicated) for the (a) $B_{1}$ QP at $(\mathrm{St}, \mathrm{Re})=(14,425)$ and $(\mathrm{b}) B_{1}$ chaotic solution at $(\mathrm{St}, \mathrm{Re})=(14,500)$. 
(a)

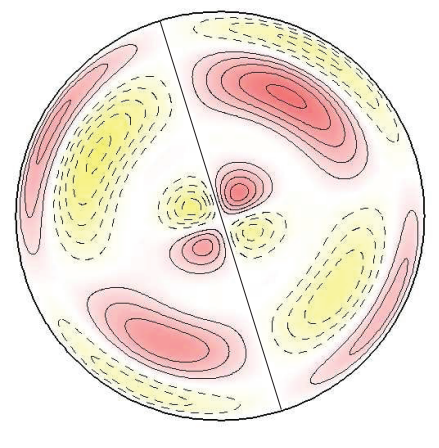

$t=t_{0}, z=-\Gamma / 4$ (b)

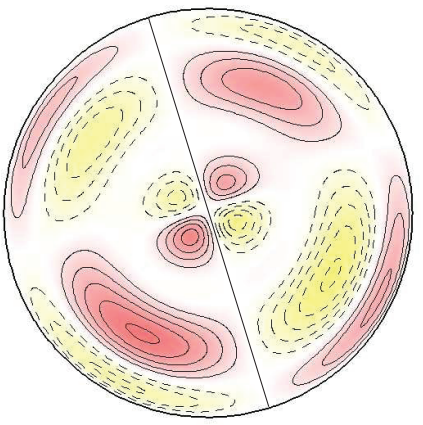

$t=t_{0}+T_{1} / 2, z=+\Gamma / 4$ (c)

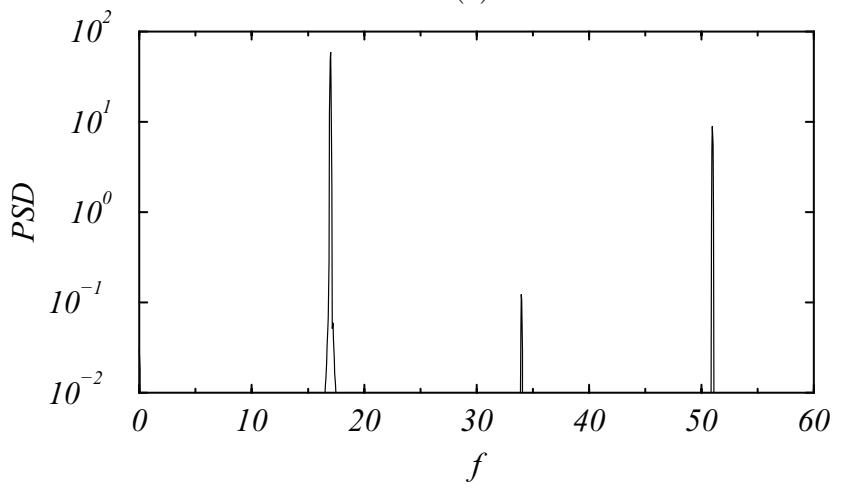

FIG. 19. (Color online) Asymmetric $B_{1}-B_{2}$ mixed mode at $(\mathrm{St}, \mathrm{Re})=(17,414)$. [(a) and (b)] Axial vorticity contours in the $z$ sections and times indicated; $t_{0}$ is at the beginning of the forcing period. Straight lines are $K_{\beta}$ reflection symmetry axes. Contour-color convention as in Fig. 5. (c) FFT of the time series of the axial velocity at midplane near the wall $(r=0.9, z=\theta=0)$.

the governing equations, the final state only has one reflection plane of symmetry. As is easily observed, this solution does not preserve the spatiotemporal $H$ symmetry: the two snapshots, which should be identical if this solution was $H$ symmetric, are rotated a factor of $\pi$. Therefore, as occurred with the $A_{2}$ modes in the previous paper, a new spatiotemporal symmetry arises: the $H$ symmetry combined with a rotation of $\pi$. Figure 19(c) represents the PSD of the time series of the axial velocity at midplane near the wall, and there is clearly a single frequency, the forcing frequency; therefore, it is a synchronous state. In contrast with all the previous solutions, the spectrum manifests even and odd harmonics of this sole frequency, which is also a clear indication of the breaking of the spatiotemporal symmetry $H$.

\section{Asymmetric bursting solution}

Starting with the asymmetric $B_{1}-B_{2}$ mixed modes and further increasing $\mathrm{Re}$, these modes become unstable and evolve towards a branch of asymmetric bursts (orange open diamonds in Fig. 14). Both asymmetric solution branches can be continued to smaller St values, and they coexist in a wide parameter region with the symmetric mixed modes and bursts, as it can be seen in Fig. 14.

(a)

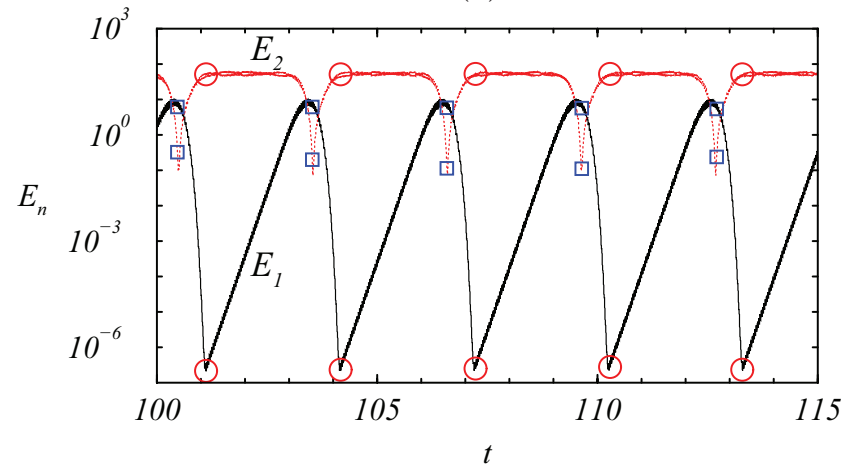

Figure 20(a) shows the time series of the energies of the asymmetric bursts at $(\mathrm{St}, \mathrm{Re})=(17,425)$. This time series is very similar to the one corresponding to the symmetric bursts in Fig. 8(a), but now the energy of the second mode is at least one order of magnitude larger than the first one, except in a very narrow temporal window where it dominates. Figure 20(b) displays the evolution of the maximum values of the axial velocity at $z=0$ near the wall. As for the symmetric bursts, in Fig. 8(b), the period measured from the axial velocity is 4 times the value obtained from the energy plot. The reason is the same as in the symmetric bursts: the solutions at two consecutive minima of the $n=2$ mode (almost pure $B_{1}$ modes) are rotated $\pi / 2$, so it takes four bursts to recover the initial state. The period measured in Fig. 20(b) is $T_{2} \approx 12.212$, very long when compared with the forcing period $T_{1}=1 / \mathrm{St}=0.0588$ : $T_{2} / T_{1} \approx 207.6$.

The bullets in Fig. 21(a) represent the Poincaré section of the asymmetric bursting solution considered, using two axial velocities measured at $z=0$ and $z=0.95$, the same section as in Fig. 10(a) from the symmetric bursts. Figure 21(b) exhibits the evolution of $S_{K_{\beta}}$ along an asymmetric burst: this parameter is constant most of the time, except for a remarkable peak and valley that coincide with the minima of the energies, also plotted in the figure. Therefore, the reflection symmetry

FIG. 20. (Color online) Asymmetric bursting solution at $(\mathrm{St}, \mathrm{Re})=(17,425)$. Time series of (a) the energy of the Fourier modes $E_{1}$ and $E_{2}$ and (b) maximum values of the near-wall axial velocity at midheight $(r=0.9, z=\theta=0)$. 
(a)

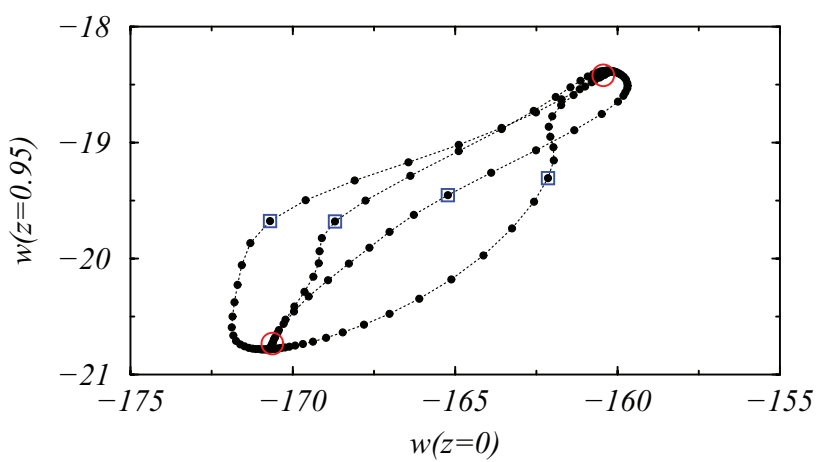

(b)

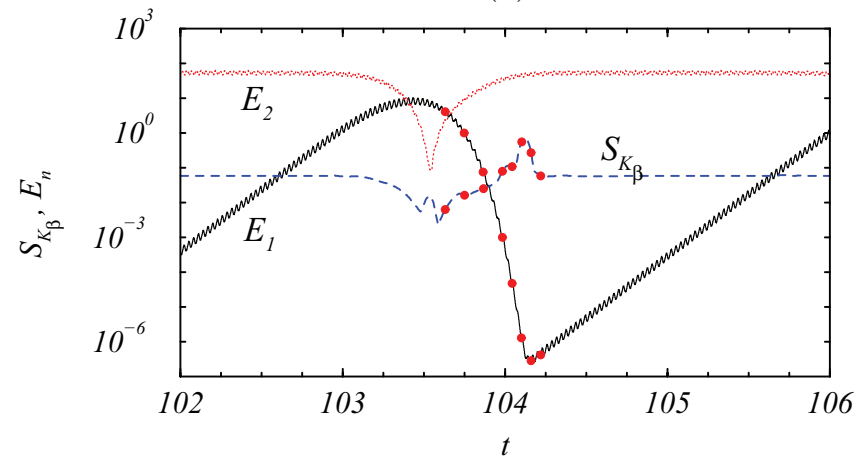

FIG. 21. (Color online) Asymmetric bursting solution at $(\mathrm{St}, \mathrm{Re})=(15.5,400)$. (a) Poincaré section (bullets) using two axial velocities measured near the wall ( $r=0.9, \theta=0$, and $z$ as indicated). The minima of $E_{1}$ (circumferences) and $E_{2}$ (open squares) indicated in Fig. 20 are also plotted. (b) Time evolution of the $K_{\beta}$ asymmetry parameter, $S_{K_{\beta}}$; symbols refer to Fig. 22.

is broken mainly when the energy of the $n=1$ mode is minimum, and the plot is similar to Fig. 10(b) for the symmetric bursts. However, the asymmetric bursts break the $K_{\beta}$ reflection symmetry in a way that differs from the symmetric bursts. Azimuthal mode one preserved reflection symmetry, while mode two slightly broke it in the symmetric bursts. The spike in the evolution of the symmetry parameter $S_{K_{\beta}}$ was due to the fact that mode one rotated $\pi / 2$ when it was very close to its minimum, but remained reflection-symmetric while rotating, as illustrated in the first row of Fig. 11. For the asymmetric bursts the azimuthal mode two behaves in the same way as for the symmetric bursts, but mode one differs enormously.
Figure 22 shows a time sequence of the first Fourier mode along the rapid decrease and about the minimum value of its energy. The eight snapshots in Fig. 22 correspond to the eight red circles in Fig. 21(b). The first five snapshots along the rapid decrease of mode one show that the symmetry line does not change and the mode is reflection-symmetric, although the shape of this mode changes remarkably. The three last snapshots, about the minimum, show that mode one breaks the reflection symmetry becoming distorted near the axis, where the pattern starts rotating, while the pattern close to the sidewall does not move at the beginning of the process (snapshot 6), and then rapidly rotating (snapshot 7 ), until, finally, the reflection
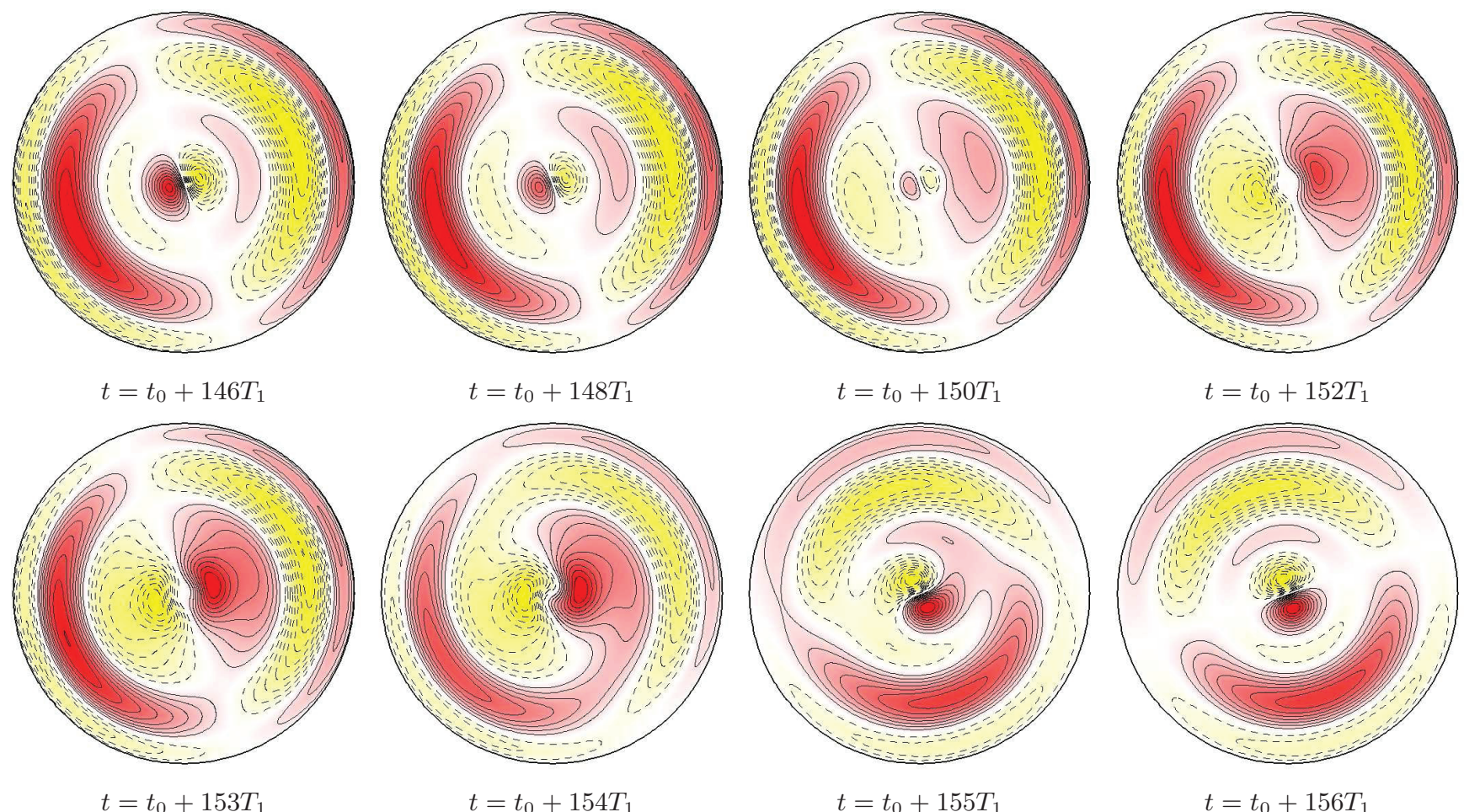

FIG. 22. (Color online) Axial vorticity contours at $z=-\Gamma / 4$ and different times of the asymmetric bursts at $(\mathrm{St}, \mathrm{Re})=(17,425) ; t_{0}$ is at the beginning of the forcing period. Only the first azimuthal Fourier component is plotted, at the points indicated by red circles in Fig. 21(b). Contour-color convention as in Fig. 5. 
(a)

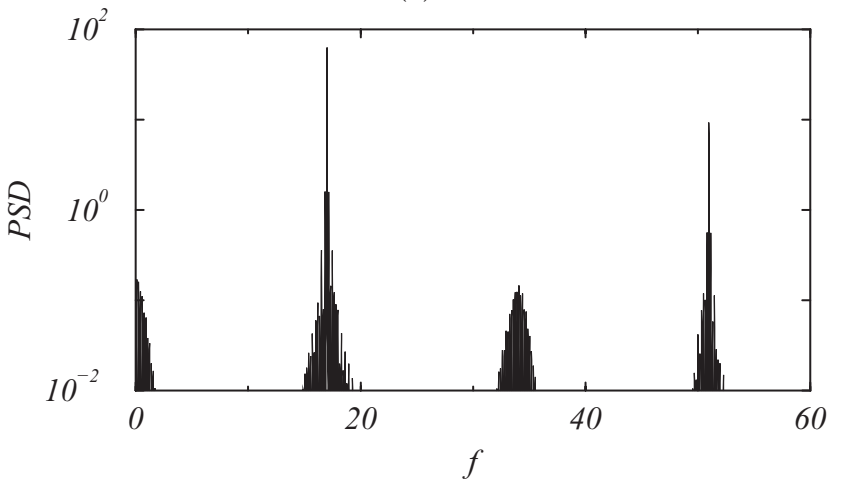

(b)

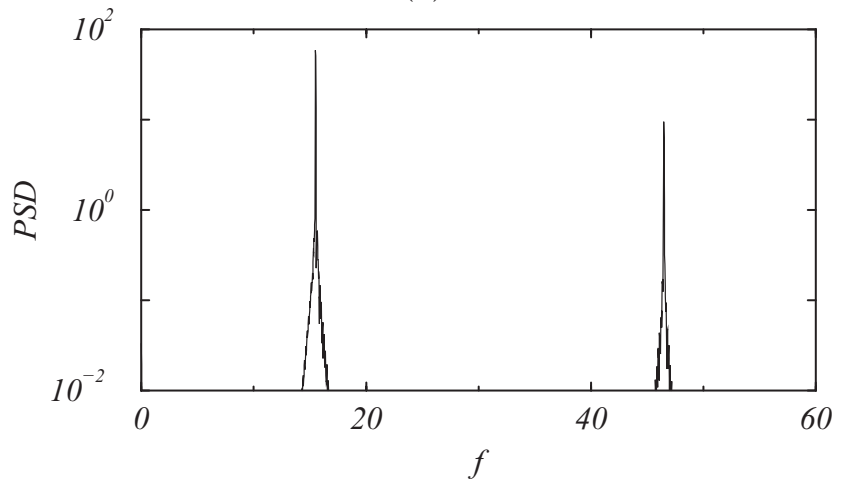

FIG. 23. FFT of the time series of (a) the asymmetric burst in Fig. 20(b) and (b) the symmetric burst in Fig. 8(b).

symmetry is restored with mode one having rotated $\pi / 2$ with respect to the starting point (snapshot 1). The distorted spiral pattern near the axis resembles the quasiperiodic mode $\mathrm{MRW}_{1}$ that bifurcates at much larger St values (see Fig. 2 ), $\mathrm{St} \approx 40.6$. This MRW $_{1}$ solution has been described in Ref. [19]. This resemblance suggests that the influence of the quasiperiodic modes could be the origin of the two families of asymmetric solutions found.

Figure 23(a) represents the PSD of the asymmetric bursting time series in Fig. 20(b), while Fig. 23(b) shows the same PSD but for the symmetric bursting solution of Fig. 8(b). The main difference is the presence of the even Fourier components of the axial velocity on the midplane for the asymmetric bursts, which were absent in the symmetric bursts, clearly manifesting that these are not $H$ symmetric. This is not surprising, because the asymmetric bursts bifurcate from the asymmetric mixed modes, as neither are $H$ symmetric. The PSDs in Fig. 23 show an enhancement of the forcing frequency peaks due to the presence of the very low frequency associated with the bursts. This enhancement is more pronounced in the asymmetric burst solutions.

\section{Irregular bursting solution}

Starting from an asymmetric bursting solution and increasing Re beyond the limits in Fig. 14, the bursts become chaotic and irregular. Figure 24(a) shows the time series of the energies of the first and second azimuthal Fourier modes after saturation, for the irregular burst at $(\mathrm{St}, \mathrm{Re})=(17,550)$. In this case, both modes acquire energies of similar order of magnitude, showing irregular bursts from time to time and chaotic temporal behavior.

In Fig. 24(b), contours of axial vorticity are depicted for $z=-\Gamma / 4$ at a particular time. See Supplemental material [25] associated with Fig. 24(b) that shows snapshots over 100 forcing periods taken at the beginning of the forcing period (a Poincaré section movie) [25]. Looking at the movie, the irregular nature of these bursts is evident. The presence of pure modes can be observed sporadically, but most of the time there is a large interaction between the different modes. This solution resembles the solutions in the bursting scenarios; however, the visits to the $B_{1}$ state are not periodic but random, and between consecutive visits the $B_{1}$ pattern rotates randomly approximately $\pm \pi / 2$. Concerning the spatial symmetries, when the solution approaches a pure or a mixed mode the corresponding spatial symmetries are observed, but otherwise the solution is largely distorted without any symmetry. For example, $S_{K_{\beta}}$ (irregular) $=0.545$ in Fig. 24(b). The Fourier transforms of the axial velocity on the midplane for this state is not shown but is very similar to the other chaotic solution, displayed in Fig. 17(b), and only possesses the odd Fourier components too. (a)

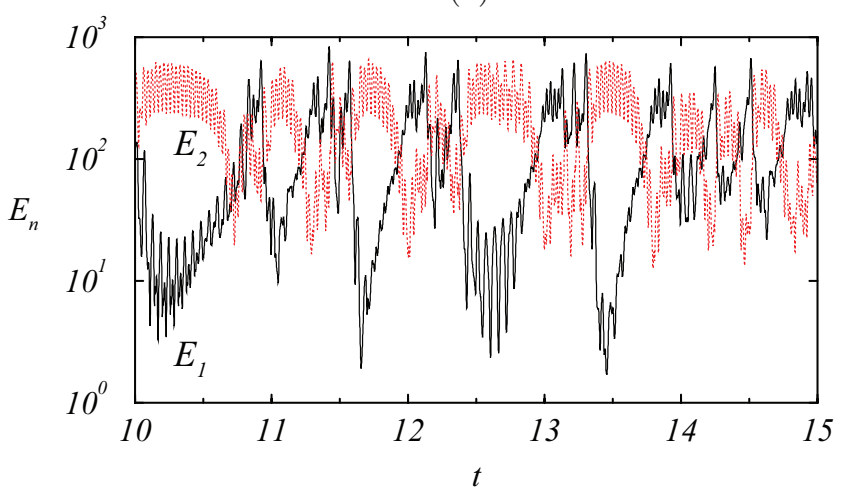

(b)

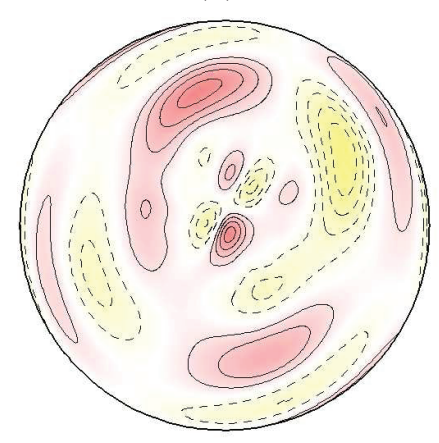

FIG. 24. (Color online) Irregular bursts at $(\mathrm{St}, \mathrm{Re})=(17,550)$. (a) Time series of the energy of the Fourier modes $E_{1}$ and $E_{2}$. (b) Axial vorticity contours at $z=-\Gamma / 4$; see Supplemental Material [25]. Contour-color convention as in Fig. 5. 
(a)

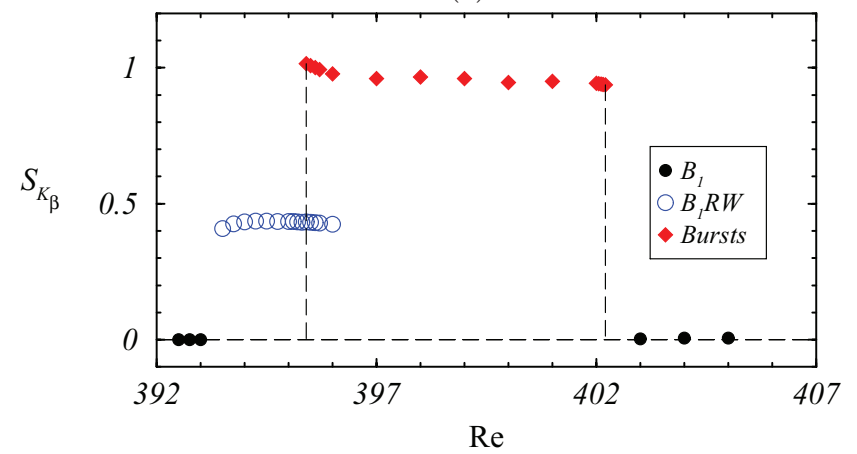

(b)

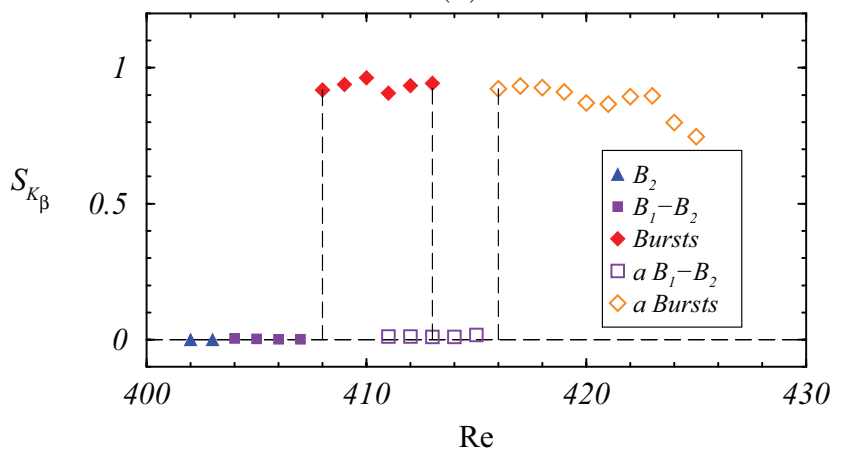

FIG. 25. (Color online) $K_{\beta}$ asymmetry parameter, $S_{K_{\beta}}$, as a function of the Reynolds number, Re, for (a) $\mathrm{St}=15.5$ and (b) $\mathrm{St}=17$.

\section{DISCUSSION}

The three-dimensional dynamics of fluid problems with spatial $\mathrm{O}(2)$ symmetry and spatiotemporal $\mathrm{Z}_{2}$ symmetry has aroused the interest of the fluid dynamics community in recent years, due to its relevance to wakes and periodically forced flows. Experiments and numerical simulations have been conducted in these flows, looking for the three-dimensional dynamics after breaking the $\mathrm{O}(2)$ symmetry $[3,4,7,8,15]$. In these different settings, the analysis of the three-dimensional dynamics has faced different setbacks. In the wake flows, the lack of enough control parameters does not allow the separated analysis of the different instability modes, which can be only observed at secondary bifurcations that become chaotic rapidly when increasing the control parameter. In other flows $[8,15]$, the dynamics are dominated by a variety of mixed modes with complicated spatiotemporal structures, even very near the onset of three-dimensional instabilities. The fluid problem analyzed in this study, a Newtonian fluid enclosed in a cylindrical cavity whose sidewall oscillates harmonically in the axial direction, solves the aforementioned problems. Special attention has been focused in the detailed analysis of the families of bifurcated solutions in a neighborhood of the first codimension- 2 point $C_{1}$ that acts as the organizing center of the dynamics at moderate frequency forcing values $(\mathrm{St} \leqslant 17)$. There exist complex dynamics with different families of mixed modes, modulated rotating waves, and bursting solutions, but, fortunately, in this problem the different bifurcations and transitions between these families can be resolved, and a detailed analysis has been presented.

The main findings are summarized in Fig. 14, and in particular in Fig. 25, showing the relationship between the different solutions obtained along two one-dimensional parameter paths at $\mathrm{St}=15.5$ and $\mathrm{St}=17$, below and above the codimension2 point $C_{1}$. Close to this bicritical point, the dynamics is dominated by two families of solutions, with dynamics governed by a very low frequency, in addition to the forcing frequency. They are the modulated rotating waves $B_{1} \mathrm{RW}$ (blue circumferences) and the symmetric bursting solutions (red solid diamonds) that appear in infinite period bifurcations. The modulated rotating waves $B_{1} \mathrm{RW}$ are present only below $C_{1}$, for $\mathrm{St}<\mathrm{St}_{c}$. Above the bicritical point $C_{1}$, a succession of three bifurcations (Base state $\rightarrow B_{2} \rightarrow B_{1}-B_{2} \rightarrow$ Bursts) occurs rapidly, resulting in bursting solutions. These rapid cascades of bifurcations are typical of fluid problems with $\mathrm{O}(2) \times \mathrm{Z}_{2}^{\mathrm{ST}}$ symmetry, and in the present problem it has been possible to resolve the details of the cascade for the first time. Close to $C_{1}$ the bifurcated solutions preserve the spatiotemporal symmetry $H$ exactly for the synchronous solutions and very approximately for the quasiperiodic solutions. However, when increasing St and Re, additional families of mixed and bursting solutions breaking the $H$ symmetry appear. These asymmetric solutions coexist and interact with the symmetric families and when increasing Re the asymmetric solutions become chaotic.

The details of the transition to chaos in systems with $\mathrm{O}(2) \times \mathrm{Z}_{2}^{\text {ST }}$ symmetry have been unraveled in this study, and hopefully the combination with the analysis of other bicritical points in these problems will help in understanding the transition to chaos in wakes and periodically forced systems. The analysis of these complex bicritical points from a theoretical dynamical system perspective has not been addressed, and it would also shed light on the understanding of these complicated processes.

\section{ACKNOWLEDGMENTS}

This work was supported by the Spanish Government Grant No. FIS2009-08821 and the Universitat Politècnica de Catalunya Grant No. FPU-UPC 250.720.
[1] C. H. K. Williamson, Phys. Fluids 31, 3165 (1988).

[2] E. Meiburg and J. C. Lasheras, J. Fluid Mech. 190, 1 (1988).

[3] C. H. K. Williamson, J. Fluid Mech. 328, 345 (1996).

[4] S. Julien, J. Lasheras, and J.-M. Chomaz, J. Fluid Mech. 479, 155 (2003).

[5] D. Barkley and R. D. Henderson, J. Fluid Mech. 322, 215 (1996).
[6] J. Robichaux, S. Balachandar, and S. P. Vanka, Phys. Fluids 11, 560 (1999).

[7] H. M. Blackburn, F. Marques, and J. M. Lopez, J. Fluid Mech. 522, 395 (2005).

[8] H. M. Blackburn and J. M. Lopez, J. Fluid Mech. 667, 336 (2011).

[9] J. C. Lasheras and E. Meiburg, Phys. Fluids A 5, 371 (1990). 
[10] D. Barkley, L. S. Tuckerman, and M. Golubitsky, Phys. Rev. E 61, 5247 (2000).

[11] F. Marques, J. M. Lopez, and H. M. Blackburn, Physica D 189, 247 (2004).

[12] M. J. Vogel, A. H. Hirsa, and J. M. Lopez, J. Fluid Mech. 478, 197 (2003).

[13] H. M. Blackburn and J. M. Lopez, J. Fluid Mech. 497, 289 (2003).

[14] H. M. Blackburn and J. M. Lopez, Phys. Fluids 15, L57 (2003).

[15] J. J. F. Leung, A. H. Hirsa, H. M. Blackburn, F. Marques, and J. M. Lopez, Phys. Rev. E 71, 026305 (2005).

[16] T. Leweke and M. Provansal, J. Fluid Mech. 280, 265 (1995).

[17] G. J. Sheard, M. C. Thompson, and K. Hourigan, J. Fluid Mech. 506, 45 (2004).
[18] H. M. Blackburn and G. J. Sheard, Phys. Fluids 22, 031701 (2010).

[19] C. Panades, F. Marques, and J. M. Lopez, J. Fluid Mech. 681, 193 (2011).

[20] I. Mercader, O. Batiste, and A. Alonso, Computers Fluids 39, 215 (2010).

[21] J. D. Crawford and E. Knobloch, Annu. Rev. Fluid Mech. 23, 341 (1991).

[22] Y. A. Kuznetsov, Elements of Applied Bifurcation Theory, 3rd ed. (Springer, Berlin, 2004).

[23] J. M. Lopez and F. Marques, J. Fluid Mech. 628, 269 (2009).

[24] S. Strogatz, Nonlinear Dynamics and Chaos (Addison-Wesley, Reading, MA, 1994).

[25] See Supplemental Material at http://link.aps.org/supplemental/ 10.1103/PhysRevE.87.043001 for a movie depicting this state. 